\title{
Abundance analysis of prime B-type targets for asteroseismology
}

\section{B6-B9.5 stars in the field of view of the CoRoT satellite ${ }^{\star, \star \star, \star \star \star}$}

\author{
E. Niemczura ${ }^{1,2}$, T. Morel $^{2,3}$, and C. Aerts ${ }^{2,4}$ \\ 1 Astronomical Institute, Wrocław University, Kopernika 11, 51-622 Wrocław, Poland \\ e-mail: eniem@astro.uni.wroc.pl \\ 2 Institute of Astronomy, Katholieke Universiteit Leuven, Celestijnenlaan 200D, 3001 Leuven, Belgium \\ e-mail: conny@ster.kuleuven.be \\ 3 Institut d'Astrophysique et de Géophysique, Université de Liège, Allée du 6 août, Bât. B5c, 4000 Liège, Belgium \\ e-mail: morel@astro.ulg.ac.be \\ 4 Department of Astrophysics, IMAPP, Radboud University Nijmegen, PO Box 9010, 6500 GL, Nijmegen, The Netherlands
}

Received 23 February 2009 / Accepted 24 May 2009

\section{ABSTRACT}

\begin{abstract}
Aims. The main goal of the ground-based seismology support program for the CoRoT mission was to obtain photometric and spectroscopic data for stars in the fields monitored by the satellite. These ground-based observations were collected in the GAUDI archive. High-resolution spectra of more than 200 B-type stars are available in this database, and about $45 \%$ of them is analysed here.

Methods. To derive the effective temperature of the stars, we used photometric indices. Surface gravities were obtained by comparing observed and theoretical Balmer line profiles. To determine the chemical abundances and rotational velocities, we used a spectrum synthesis method, which consisted of comparing the observed spectrum with theoretical ones based on the assumption of LTE. Results. Atmospheric parameters, chemical abundances, and rotational velocities were determined for 89 late-B stars. The dominant species in their spectra are iron-peak elements. The average Fe abundance is $7.24 \pm 0.45$ dex. The average rotational velocity is $126 \mathrm{~km} \mathrm{~s}^{-1}$, but there are 13 and 20 stars with low and moderate $V \sin i$ values, respectively. The analysis of this sample of 89 late B-type stars reveals many chemically peculiar (CP) stars. Some of them were previously known, but at least 9 new CP candidates, among which at least two HgMn stars, are identified in our study. These CP stars as a group exhibit $V \sin i$ values lower than the stars with normal surface chemical composition.
\end{abstract}

Key words. stars: abundances - stars: atmospheres - stars: chemically peculiar

\section{Introduction}

In preparation for the CoRoT space mission (Auvergne et al. 2009), an extensive ground-based photometric and spectroscopic campaign of the stars located in the field of view of the satellite was performed. All of these data were collected in the Groundbased Asteroseismology Uniform Database Interface (GAUDI, Solano et al. 2005). This catalogue contains a vast number of spectra of B-type stars, among others.

Only a few investigations of B stars in the GAUDI archive have been completed. Neiner et al. (2005) discovered 17 new Be stars in the GAUDI database. Frémat et al. (2006) determined the fundamental parameters (spectral type, temperature, gravity, $V \sin i$ ) of the 64 Be stars in the seismology fields of CoRoT by

\footnotetext{
* Atmospheric parameters and abundance analysis results are only available in electronic form at the CDS via anonymous ftp to cdsarc.u-strasbg.fr (130.79.128.5) or via

http://cdsweb.u-strasbg.fr/cgi-bin/qcat?J/A+A/506/213

$\star \star$ The CoRoT space mission was developed and is operated by the French space agency CNES, with participation of ESA's RSSD and Science Programmes, Austria, Belgium, Brazil, Germany, and Spain.

$\star \star \star$ Tables 1 and 2 are only available in electronic form at http://www. aanda.org
}

performing a detailed modelling of the stellar spectra. Lefever et al. (2006) and Lefever (2007) estimated the fundamental stellar parameters, i.e., effective temperatures, surface gravities, luminosities, $\mathrm{He}$ and $\mathrm{Si}$ abundances, and projected rotational velocities of a subsample of B stars in GAUDI. They used line-profile fitting methods based on non-LTE line-formation predictions for unified atmospheres with winds. Morel \& Aerts (2007) presented an abundance study of two early B-type targets for the asteroseismology programme of the CoRoT mission.

To provide a homogeneous high-precision determination of the fundamental parameters and abundances of the prime B-type targets of the CoRoT space mission, Morel et al. (2006; Paper I) performed a pilot study of nine bright prototypical early-B $\beta$ Cephei stars. This study was based on Kurucz (1993) atmosphere models and non-LTE modelling techniques, as well as a time series analysis of high-quality spectroscopic data assembled for these stars primarily for spectroscopic mode identification. In a follow-up study, Morel et al. (2008) increased the sample to 20 stars. These studies unexpectedly discovered a population of nitrogen-rich and boron-depleted, yet intrinsically slowly-rotating early-B stars (see also, e.g., Gies \& Lambert 1992). A higher incidence of chemical peculiarities was also found in the stars for which a weak magnetic field was detected. 
Although this needs to be confirmed by analysing larger samples, this suggests that magnetic phenomena may be important in altering the photospheric abundances of early B dwarfs, even for weak surface field strengths.

As pointed out by Morel \& Aerts (2007), there is a large amount of spectroscopic data of B-type stars in the GAUDI archive. Most of these stars are late B-type objects. In this paper, we present a homogeneous study of 89 B6-B9.5 stars in the field of view of the CoRoT mission. The chemical abundances that we derive here provide useful additional information for the interpretation of the CoRoT data.

The atmospheres of late-B main-sequence stars are relatively simple to model. There is no convection, stellar winds are very weak, and for the majority of these objects the microturbulence is very low. In these conditions, diffusion can easily occur. A significant number of late-B stars exhibit spectroscopic signatures of these microscopic transport processes (e.g., Hempel \& Holweger 2003; Folsom et al. 2007). The investigation of late-B star atmospheres will help us to understand these physical phenomena. It will provide valuable information about the behaviour of an element influenced by both gravitation and radiation, leading to a variety of abundance patterns even in nonmagnetic stars. Our goal is to investigate these effects for a large number of stars.

This paper is organised as follows. In Sect. 2, we describe the spectroscopic observations used in the abundance analysis. In Sect. 3, we present the method for determining the atmospheric parameters, abundance patterns and projected rotational velocities of the objects. The sources of possible errors in the chemical abundances are described in Sect. 4. The measured $V \sin i$ values and elemental abundances are discussed in Sects. 5 and 6 , respectively. Section 7 contains a detailed discussion of the chemical abundances of known and newly discovered chemically peculiar stars. Conclusions and future prospects are given in Sect. 8. Reliability tests of our abundance analysis method are presented in Appendix A.

\section{Spectroscopic observations}

We analyse the high-resolution $(R \cong 40000-50000)$ spectroscopic observations of B-type stars obtained with the ELODIE and FEROS spectrographs (see Table 1). The ELODIE spectrograph (Baranne et al. 1996) was attached to the $1.93 \mathrm{~m}$ telescope at the Observatoire de Haute-Provence, France. The FEROS spectrograph (Kaufer et al. 2000) was installed on the $1.52 \mathrm{~m}$ and $2.2 \mathrm{~m}$ telescopes at La Silla, Chile. Both instruments are cross-dispersed, fibre-fed echelle spectrographs. Typical signal-to-noise ratios of the spectra range from 100 to 150 at $5500 \AA$. The wavelength intervals covered are 3900-6800 ̊ and 3800-9100 ̊ for ELODIE and FEROS, respectively. For the details of the data reduction, we refer to Solano et al. (2005). In our initial sample, we have 160 FEROS spectra and 62 ELODIE spectra of B-type stars. Only the spectroscopic observations of the B6-B9.5 stars available in the GAUDI archive are analysed in this paper. We also excluded Be stars, supergiants and spectroscopic binaries for which the lines of two stars were visible in the spectra. Basic information about the analysed stars, including spectral type, name of the spectrograph, observation date in UT, $V$ magnitude, interstellar reddening, indications of binarity, and abundance determinations in the literature are given in Table $1^{1}$.

A comparison between the Balmer line profiles for stars with both FEROS and ELODIE spectra in GAUDI showed that the

1 Tables 1 and 2 are only available in the electronic form. wings are far less pronounced in the latter (see Lefever 2007), whereas good agreement was found with ELODIE spectra taken from the archives ${ }^{2}$. Although the origin of the problem affecting the ELODIE spectra in GAUDI is unclear (but probably related to the data reduction), using these data would produce spuriously low gravity estimates. We therefore only used archival ELODIE spectra in our analysis. All the spectra were carefully normalised by us using standard IRAF procedures. This was found to be necessary to guarantee high-precision estimates of the fundamental parameters, especially the gravity. The extraction of Balmer line profiles from echelle spectra can be problematic, because in many cases the wings extend beyond the spectral coverage of a single order in the echellogram or the line is situated at the edge of the order. This is the situation for the FEROS and ELODIE spectrographs. In these cases, the proper setting of the line intensities depends not only on the continuum placement process but also on the data reduction (flat-fielding, order connections). For this reason, all Balmer lines suitable for determining the surface gravity were analysed (see Sect. 3). We found that there is a high degree of consistency between the $\log g$ derived from different lines, typically $\Delta \log g<0.2$.

\section{Method of analysis}

To perform an abundance analysis, one needs to develop an atmospheric model of the star, which requires the knowledge of its effective temperature, surface gravity and metallicity. To determine the initial stellar effective temperatures and surface gravities, we applied photometric and spectroscopic methods, respectively.

The photometric data were taken from the catalogues accessible in the GCPD database (Hauck \& Mermilliod 1998) ${ }^{3}$ and from the GAUDI archive. For every star, the average Strömgren and Geneva photometric values were computed.

The effective temperatures were determined on the basis of $u v b y \beta$ photometry using the UVBYBETA code written by Moon \& Dworetsky (1985) and corrected by Napiwotzki et al. (1993). For all stars for which Geneva photometry is available, we used codes and calibrations performed by Künzli et al. (1997). These calibrations can be applied to B, A, and F stars with luminosity classes V-III and are based on LTE Kurucz atmosphere models. The comparison of the $T_{\text {eff }}$ values determined from both photometric systems are shown in Fig. 1, where $T_{\text {eff }}(\mathrm{MN} 1)$ and $T_{\text {eff }}(\mathrm{MN} 2)$ refer to the effective temperatures determined from the $(b-y)_{0}$ and/or $c_{0}$ indicators, and from the $[u-b]$ indicator, respectively. The most significant discrepancies between the results of Strömgren and Geneva calibrations are found for stars with effective temperatures lower than $11000 \mathrm{~K}$. For a few stars with higher temperature, the differences also exceed $500 \mathrm{~K}$. This may be caused by incorrect values of photometric indices, the adopted colour excess, or the chemical peculiarity of the stars. We checked how aforementioned effects influence our $T_{\text {eff }}$ determinations.

As mentioned above, we used Strömgren photometry from two archives. For all the stars with both datasets available, we calculated the effective temperatures independently. The differences between the two sets of temperatures are typically lower than $200 \mathrm{~K}$. In only two cases (HD 44354 and HD 48497), the $T_{\text {eff }}$ values obtained from the $(b-y)_{0}$ index differ by about $500 \mathrm{~K}$. In the case of $T_{\text {eff }}$ determined from the $[u-b]$ index, all the differences are below $500 \mathrm{~K}$, typically close to $200 \mathrm{~K}$. These

\footnotetext{
2 http://atlas.obs-hp. fr/elodie/

3 http://obswww . unige.ch/gcpd/gcpd.html
} 

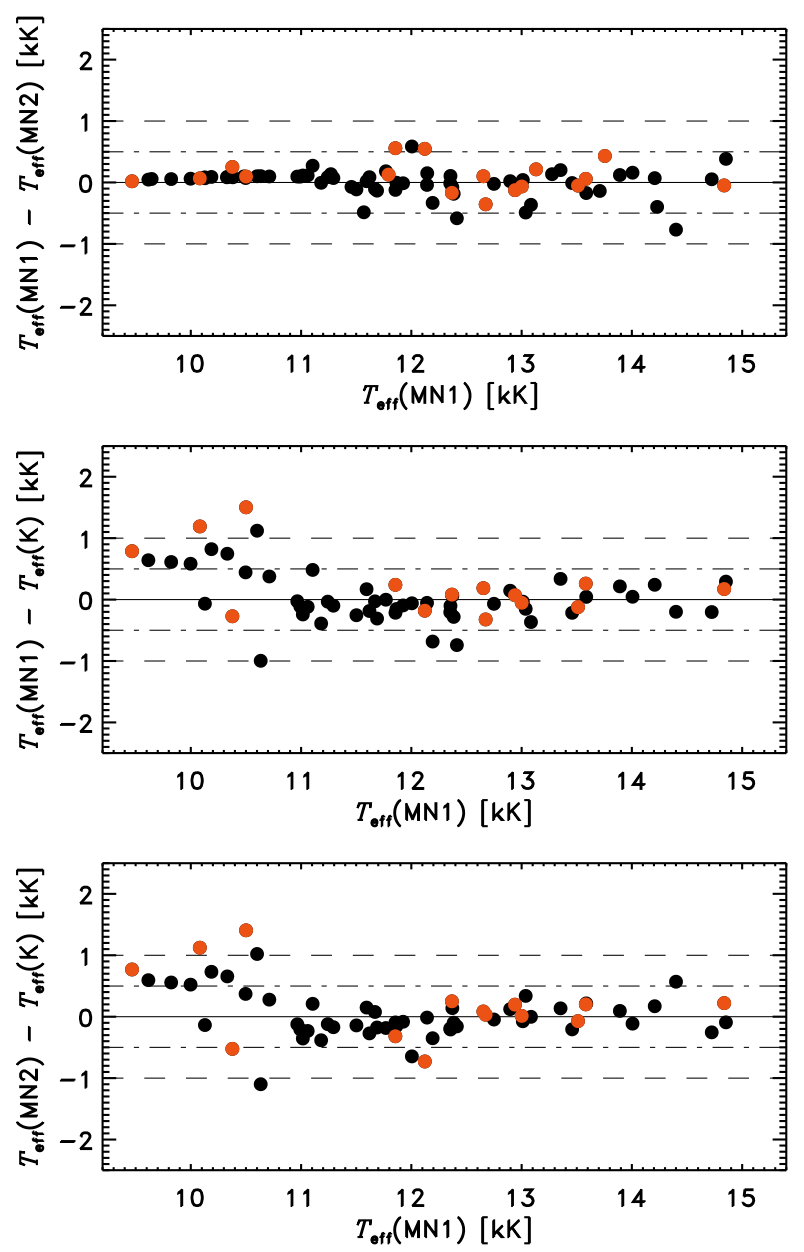

Fig. 1. Comparison between the effective temperatures calculated from Strömgren and Geneva photometry. The $T_{\text {eff }}(\mathrm{MN} 1)$ and $T_{\text {eff }}(\mathrm{MN} 2)$ symbols indicate $T_{\text {eff }}$ values determined by the two methods evaluated by Napiwotzki et al. (1993), $T_{\text {eff }}(\mathrm{K})$ corresponds to effective temperatures obtained from Geneva photometry (Künzli et al. 1997). Red dots denote confirmed or suspected CP stars.

discrepancies are caused by incorrect values of photometric indices.

The colour excess values were determined as in Netopil et al. (2008). The colours of $U B V$ photometry were taken from the SIMBAD database. In some cases, the $U B V$ colours were unavailable. For these stars, the colour excesses $E(B-V)$ were determined from $E(b-y)$ values, calculated by the UVBYBETA code. The majority of our objects have $E(B-V)$ lower than $0.1 \mathrm{mag}$. All the stars for which the discrep-

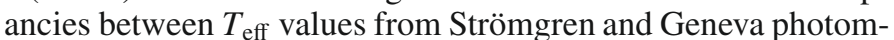
etry are higher than $500 \mathrm{~K}$ have low colour excesses, $E(B-V)<$ 0.05 mag. The $E(B-V)$ values are reported in Table 1 . In all cases where errors in colour indices are available, we determined the value of errors in colour excesses $E(B-V)$. In all cases, these errors are about 0.02 mag.

Among the objects with the highest discrepancies between the temperatures determined from different photometry, only five can be chemically peculiar (HD 47759, HD 168932, HD 46340, HD 168202, HD 49713). In Fig. 1, the suspected and known chemically peculiar stars discussed in Sect. 7 are shown with different colours. As can be seen, the scatter for the sample of normal and peculiar stars is similar. The temperature calibrations of CP stars are discussed in detail by Netopil et al. (2008). If

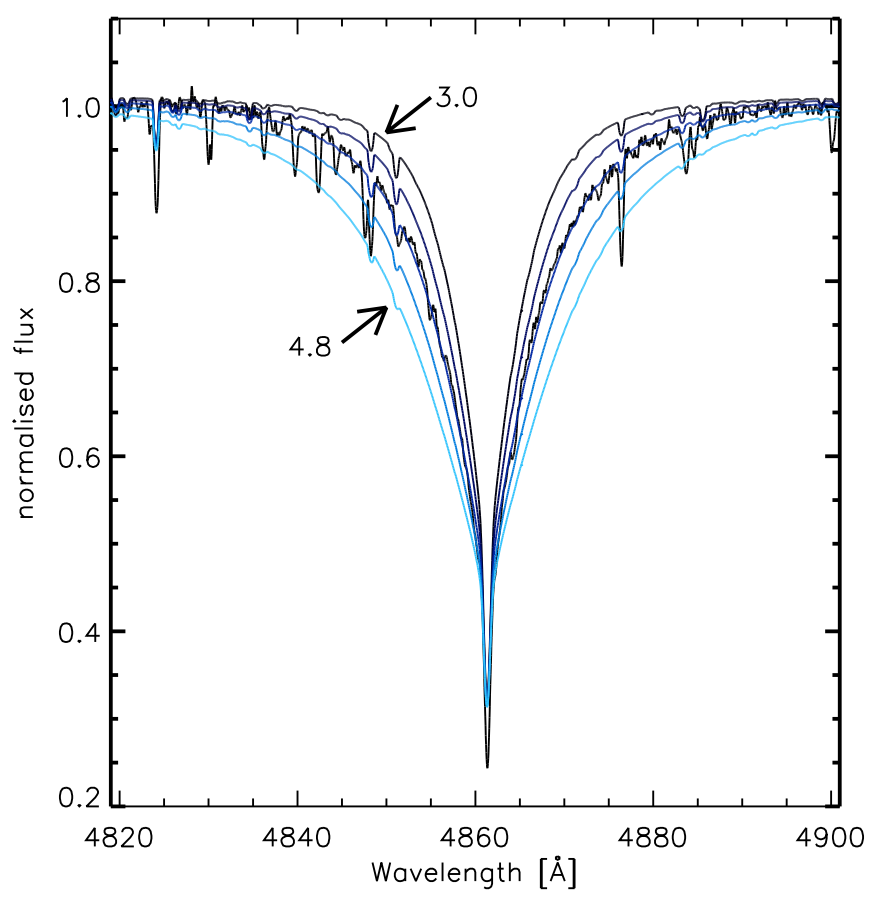

Fig. 2. Surface gravity determination from Balmer lines. The observed $\mathrm{H} \beta$ profile of HD 46886 is shown together with synthetic profiles obtained for different $\log g$ values (from 3.0 to 4.6 dex, with a step of 0.4 dex) and $T_{\text {eff }}=12900 \mathrm{~K}$.

the discrepancies between $T_{\text {eff }}$ obtained from $u v b y \beta$ and Geneva photometry exceeded $1000 \mathrm{~K}$, we adopted the average value from the two methods presented by Napiwotzki et al. (1993).

Once the average photometric effective temperature was obtained, the surface gravity was derived by comparing the observed Balmer profiles with theoretical ones. Four observed Balmer lines, $\mathrm{H} \alpha, \mathrm{H} \beta, \mathrm{H} \gamma$, and $\mathrm{H} \delta$, were used in the case of slowly rotating stars. Considering the uncertainties in the continuum normalisation, for rapidly rotating stars we used only $\mathrm{H} \alpha$ and $\mathrm{H} \beta$, because of the relatively small number of other nearby lines. The adopted average values of effective temperatures and surface gravities are shown in Table 2. As an example, the observed $\mathrm{H} \beta$ profile of $\mathrm{HD} 46886$ is shown together with synthetic profiles obtained for different $\log g$ values and $T_{\text {eff }}=12900 \mathrm{~K}$ in Fig. 2.

For the atmospheric parameters obtained, the atmospheric models were computed with the line-blanketed LTE ATLAS9 code (Kurucz 1993), which treats line opacity with opacity distribution functions (ODFs). The assumed microturbulence is $2 \mathrm{~km} \mathrm{~s}^{-1}$. The synthetic spectra were computed with the SYNTHE code (Kurucz 1993). Both codes, ATLAS9 and SYNTHE were ported under GNU Linux by Sbordone et al. (2005) and are available online ${ }^{4}$. The stellar line identification and the abundance analysis were performed on the basis of the VALD line lists (Kupka et al. $2000^{5}$, and references therein). For some Mn features in the case of $\mathrm{HgMn}$ stars and for one Ga line (Ga II 16334), the atomic data from Castelli \& Hubrig $(2004)^{6}$ were adopted.

\footnotetext{
4 wwwuser.oat.ts.astro.it/atmos/

5 http://ams.astro.univie.ac.at/vald/

${ }^{6}$ http://wwwuser. oat.ts.astro.it/castelli/grids.html
} 


\subsection{Description of the spectrum synthesis method}

Our analysis follows the methodology presented in Niemczura \& Połubek (2006) and relies on an efficient spectral synthesis based on a least squares optimisation algorithm (Takeda 1995; Bevington 1969). This method allows for the simultaneous determination of various parameters involved with stellar spectra and consists of the minimisation of the deviation between the theoretical flux distribution and the observed one. The synthetic spectrum depends on the stellar parameters, such as effective temperature $T_{\text {eff }}$, surface gravity $\log g$, rotational velocity $V \sin i$, microturbulence $\xi$, radial velocity $V_{\mathrm{r}}$, and the relative abundances of the elements $\varepsilon(\mathrm{El})$, where $\mathrm{El}$ denotes the individual element. The first two parameters and the microturbulent velocity were not determined during the iteration process but were considered as input parameters. For the majority of stars, $\xi=2 \mathrm{~km} \mathrm{~s}^{-1}$ was adopted. For only a few chemically peculiar stars, a lower value of $\xi$ was assumed $(\xi=$ $0 \mathrm{~km} \mathrm{~s}^{-1}$ for HD 168932, HD 179761, HD 46886, HD 49886, and $\xi=1 \mathrm{~km} \mathrm{~s}^{-1}$ for HD 49481, HD 50251). All the other abovementioned parameters can be determined simultaneously because they produce detectable and different spectral signatures. The $V \sin i$ values were determined by comparing the shapes of metal line profiles with the computed profiles, as shown in Gray (2005). The limb darkening coefficient, $\epsilon$, was set to be 0.6 (see Gray 2005, Sect. 18). Wade \& Rucinski (1985) and Diaz-Cordoves et al. (1995) discussed the problem of linear and non-linear limb-darkening coefficients for LTE model atmospheres. According to them, for late B-type stars the linear limb-darkening coefficient is about 0.4 . Assuming $\epsilon=0.6$ (typical of grey atmospheres) will have a small effect on the derived projected rotational velocities, but the differences will be comparable with the standard deviations $1 \sigma$. The theoretical spectrum was fitted to the normalised observed one and the continuum was objectively drawn by connecting the highest points of the analysed spectrum part. As an example of the spectral synthesis method, we show in Fig. 3 a comparison between theoretical and observed spectra for the first 12 iteration steps of an illustrative spectral range of HD 46886. In Fig. 4, variations in $V \sin i$, $\log \varepsilon(\mathrm{Fe}), \log \varepsilon(\mathrm{Mn})$, and rms (root mean square, the goodnessof-fit parameter) corresponding to the analysis of the same spectral part are shown. For the adopted input values, the solution is typically reached after 8 iterations. The program stops if the values of the determined parameters remain the same within $2 \%$ for three consecutive steps. The reliability of our method is discussed in Appendix A.

After estimating the atmospheric parameters $T_{\text {eff }}$ and $\log g$ using the methods described above, the analysis of every star proceeded in a few steps:

1. Selection of the spectral parts designated for the analysis. The length of the chosen part depends mainly on the projected rotational velocity of the star. In the case of slowly and moderately rotating objects $\left(V \sin i<80 \mathrm{~km} \mathrm{~s}^{-1}\right)$, short sections covering only a few spectral lines were investigated. For the stars with $V \sin i \gtrsim 80 \mathrm{~km} \mathrm{~s}^{-1}$, the spectral lines are significantly blended and the continuum normalisation is difficult. For these stars, we used broader spectral ranges normalised by comparison with theoretical spectra (if necessary).

2. Line identification in the chosen part. In the case of blended lines, more than one chemical element influences the line profile. The choice of the elements to be included in the analysis proceeded in a few steps. First, we calculated the spectra individually for each element, omitting the other species. All

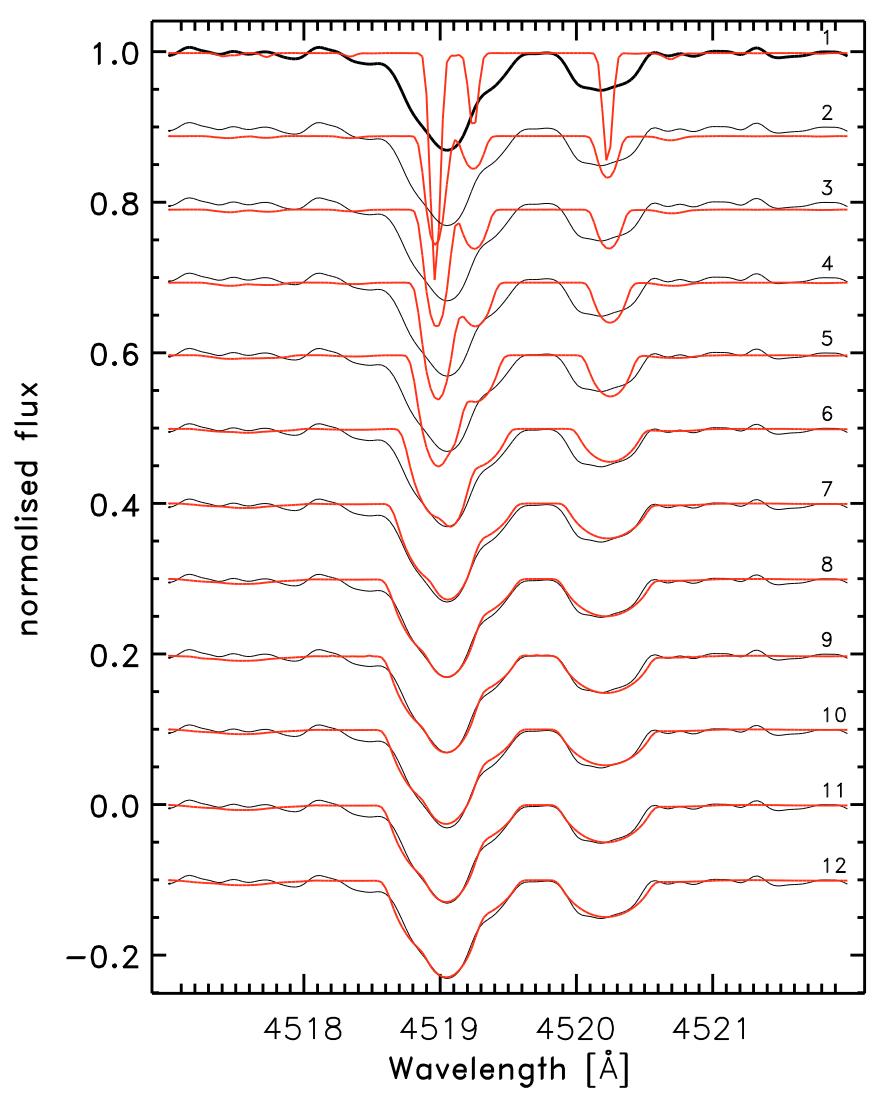

Fig. 3. Comparison between the observed and synthetic spectra for the first 12 iteration steps in the case of HD 46886. The input values are $V \sin i=0 \mathrm{~km} \mathrm{~s}^{-1}, \log \varepsilon(\mathrm{Fe})=7.50$, and $\log \varepsilon(\mathrm{Mn})=7.39$, whereas the output values are $V \sin i=23 \mathrm{~km} \mathrm{~s}^{-1}, \log \varepsilon(\mathrm{Fe})=6.61$ and $\log \varepsilon(\mathrm{Mn})=$ 7.29. These values are close to the final results obtained for HD 46886 on the basis of the whole spectrum (see Table 2).

these spectra were then normalised to the continuum level. This step was performed for every star separately, taking into account the individual stellar atmospheric parameters obtained, and the estimated rotation and radial velocity values. Second, for each element we calculated the line depths in all analysed spectral ranges. Only the most important elements producing spectral lines of significant depth were considered further.

3. For the determination of $V_{\mathrm{r}}, V \sin i$, and abundances for the adopted $T_{\text {eff }}, \log g$, and $\xi$, the method described above was used. We adjusted the abundances to determine the closest match between the calculated and observed spectral lines.

4. The determination of mean values of $V_{\mathrm{r}}, V \sin i$, and abundances of all the chemical elements considered for a given star. These values are listed in Table 2 .

5. Estimation of errors in the obtained abundances was performed.

The results of the analysis are given in Table 2. For each star, the atmospheric parameters, i.e., effective temperature, surface gravity, rotation velocity $(V \sin i)$, and the determined abundances of the elements are shown. The abundances of the chemical elements, $\log \varepsilon(\mathrm{El})(\mathrm{El}$ denotes the individual element) are given with the standard deviations $(\sigma)$ and the number of lines used in the analysis. 


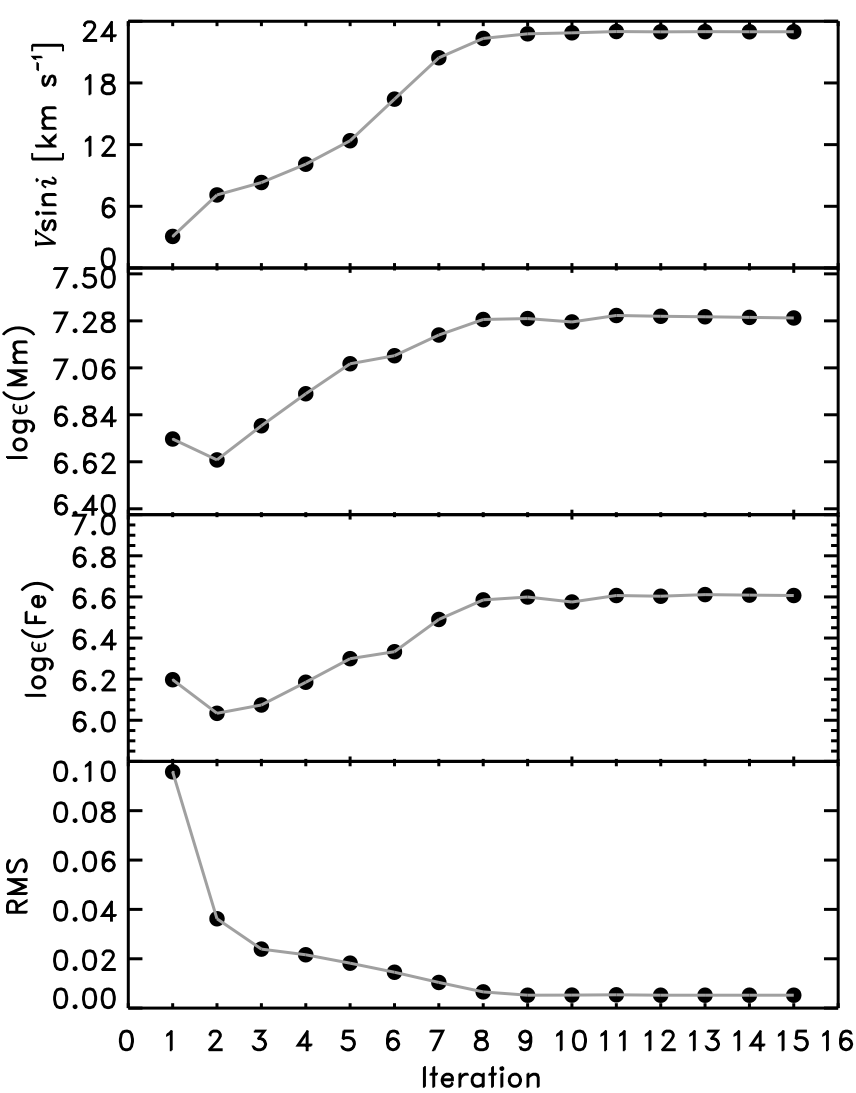

Fig. 4. Variations in $V \sin i, \log \varepsilon(\mathrm{Fe}), \log \varepsilon(\mathrm{Mn})$, and rms for the case of HD 46886 and the spectral range shown in Fig. 3.

\section{Discussion of errors}

Besides the line-to-line scatter, the derived abundances are affected by errors related to a number of sources of uncertainty, including the adopted atmospheric models and atomic data, most importantly the oscillator strengths. Additionally, the quality of the observed spectra and their normalisation have an influence on the chemical abundances obtained and other stellar parameters. We did not take into account non-LTE effects, which can be a significant source of errors in the case of some species, especially the light elements. For this reason, we did not use the O I 7771-5 A triplet for the determination of the oxygen abundance (Paunzen et al. 1999). The non-LTE effects for B-type stars are discussed in detail by Hempel \& Holweger (2003). They analysed the abundances of several light elements (He, $\mathrm{C}, \mathrm{O}, \mathrm{Ne}, \mathrm{Mg}, \mathrm{Si}, \mathrm{Ca}$ ), $\mathrm{Fe}$, and two heavy elements ( $\mathrm{Sr}, \mathrm{Ba})$ in 27 bright B5-B9 main-sequence stars. According to their results, departures from LTE are insignificant for $\mathrm{Fe}$ and $\mathrm{Mg}$. For $\mathrm{C}, \mathrm{Ne}$, and $\mathrm{Ca}$, the non-LTE corrections are below 0.2-0.3 dex. The highest corrections were obtained for $\mathrm{O}$ (in the case of the O I 7771-5 A triplet) and for Sr. For the latter, the corrections are smaller for higher temperatures. For $\mathrm{Si}$, the departures from LTE

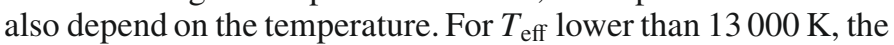
corrections are below 0.2 dex, but for higher temperatures they can exceed 0.5 dex. The effects of departures from LTE on nitrogen and barium are discussed by Przybilla \& Butler (2001) and Gigas (1988), and for Vega-like stars typically amount to -0.2 and +0.3 dex, respectively.

The errors in the adopted stellar parameters (effective temperature, surface gravity, and microturbulence) also contribute to the uncertainties in the abundances. The systematic errors
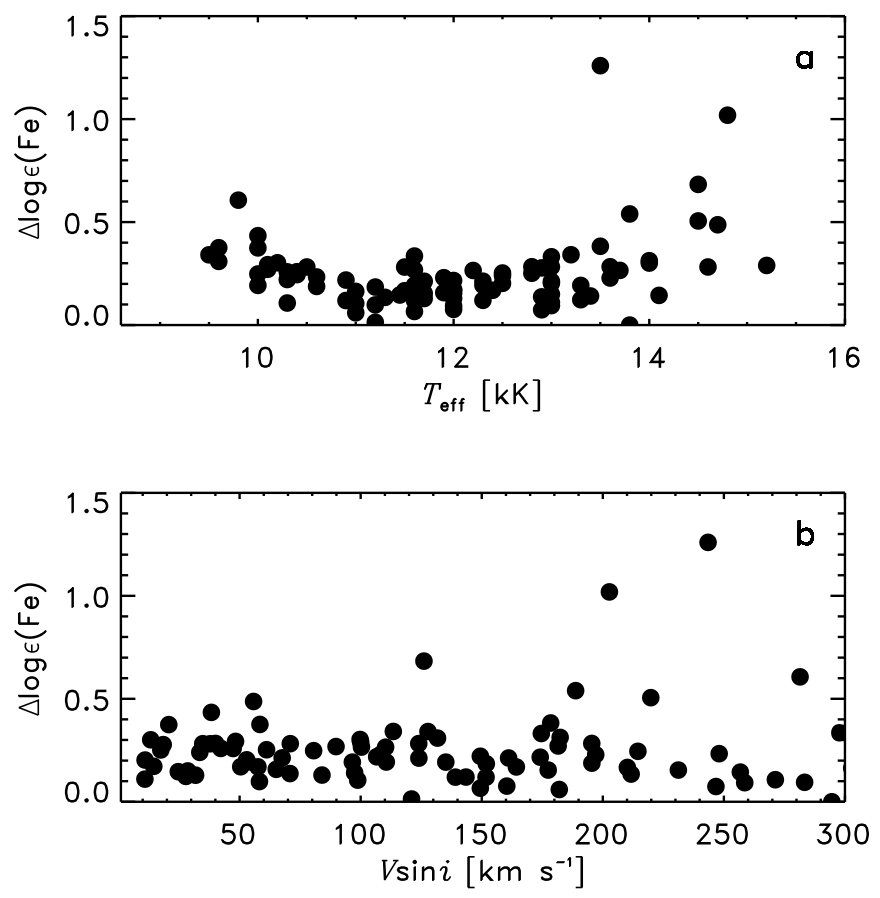

Fig. 5. Uncertainties in the Fe abundances caused by a change of $T_{\text {eff }}$ by $10 \%$, as a function of a) effective temperature and b) rotation velocity.

arising from the above parameters can be estimated directly by recalculating the abundances for slightly different values of $T_{\text {eff }}, \log g$, and $\xi$ than the adopted ones. We redetermined the mean abundances allowing for a change in $T_{\text {eff }}$ by $10 \%, \log g$ by $0.1 \mathrm{dex}$, and $\xi$ by $1 \mathrm{~km} \mathrm{~s}^{-1}$.

In Figs. 5-7, the variations in the Fe abundance caused by a change in $T_{\text {eff }}, \log g$, and $\xi$ are shown. Iron lines are present in the spectrum of every star and for the majority of them it is the element with the highest number of lines. The uncertainties in Fe caused by errors in the effective temperature are in most cases lower than 0.3 dex. Only for four stars (HD 176158, HD 45760, HD 50751, HD 56006), $\Delta \log \varepsilon(\mathrm{Fe})$ exceeds 0.5 dex. In the case of HD 45760, HD 50751, and HD 56006, the large change in abundance is caused by the high rotational velocity of these stars $\left(V \sin i>200 \mathrm{~km} \mathrm{~s}^{-1}\right)$, which can cause problems in the continuum placement. The star HD 176158 is also a rapid rotator $\left(V \sin i>100 \mathrm{~km} \mathrm{~s}^{-1}\right)$, and in all these cases the Fe abundances were determined only from 1 or 2 lines. If we ignore these four objects, there is no trend between $\Delta \log \varepsilon(\mathrm{Fe})$ produced by changes in $T_{\text {eff }}$ or $V \sin i$. The sensitivity in the abundances to errors in $T_{\text {eff }}$ depends strongly on the element considered. For more discussion, see Appendix A.

The variations in the $\mathrm{Fe}$ abundance caused by errors in the surface gravity of $0.1 \mathrm{dex}$ are in most cases lower than $0.1 \mathrm{dex}$. There is no correlation between $\Delta \log \varepsilon(\mathrm{Fe})$ and $\log g$. For only two stars with moderate rotation, the error in the Fe abundance is larger than 0.1 dex (HD 168932 and HD 181761). For all the other cases, $V \sin i>100 \mathrm{~km} \mathrm{~s}^{-1}$. There is some evidence of a small trend between $\Delta \log \epsilon(\mathrm{Fe})$ and $V \sin i$. For higher $V \sin i$ values, the scatter in Fig. $6 \mathrm{~b}$ is larger.

The change in $\xi$ by $1 \mathrm{~km} \mathrm{~s}^{-1}$ causes variations in the Fe abundances lower than 0.2 dex. For only three fast rotating stars (HD 171931, HD 49643, HD 49711), the uncertainties in Fe slightly exceed 0.2 dex. Here we compare only the average $\mathrm{Fe}$ abundances obtained from the analysis of all iron lines 

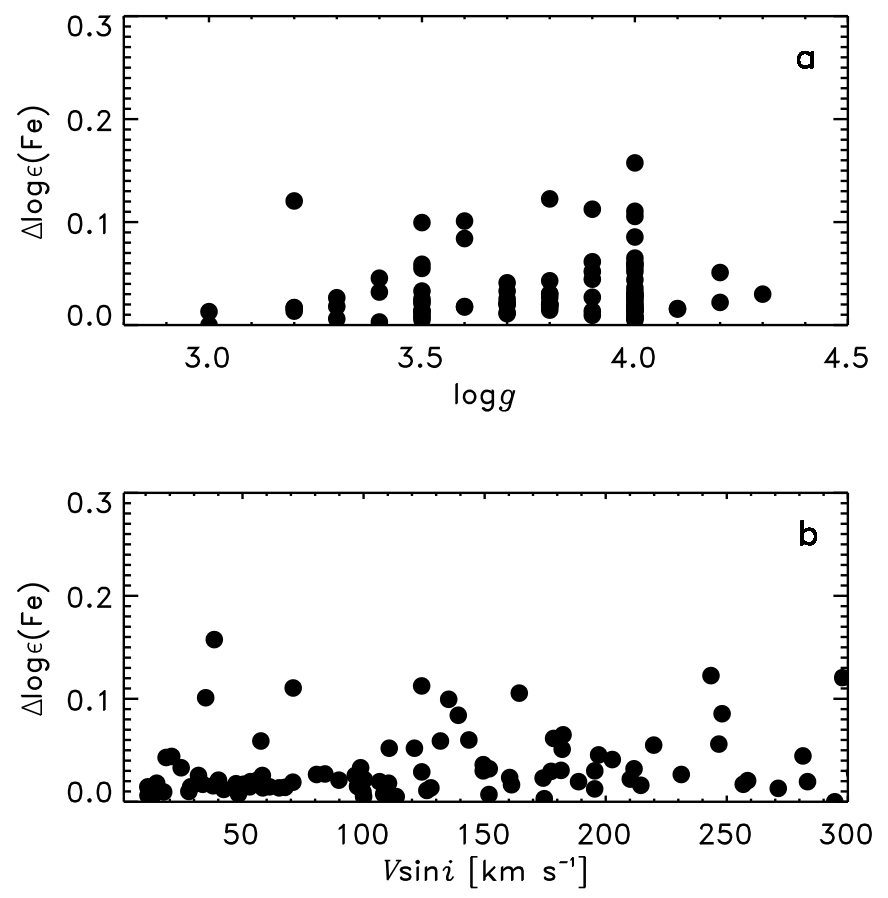

Fig. 6. Uncertainties in the Fe abundances caused by a change of $\log g$ by 0.1 dex, as a function of a) surface gravity and $\mathbf{b}$ ) rotation velocity.
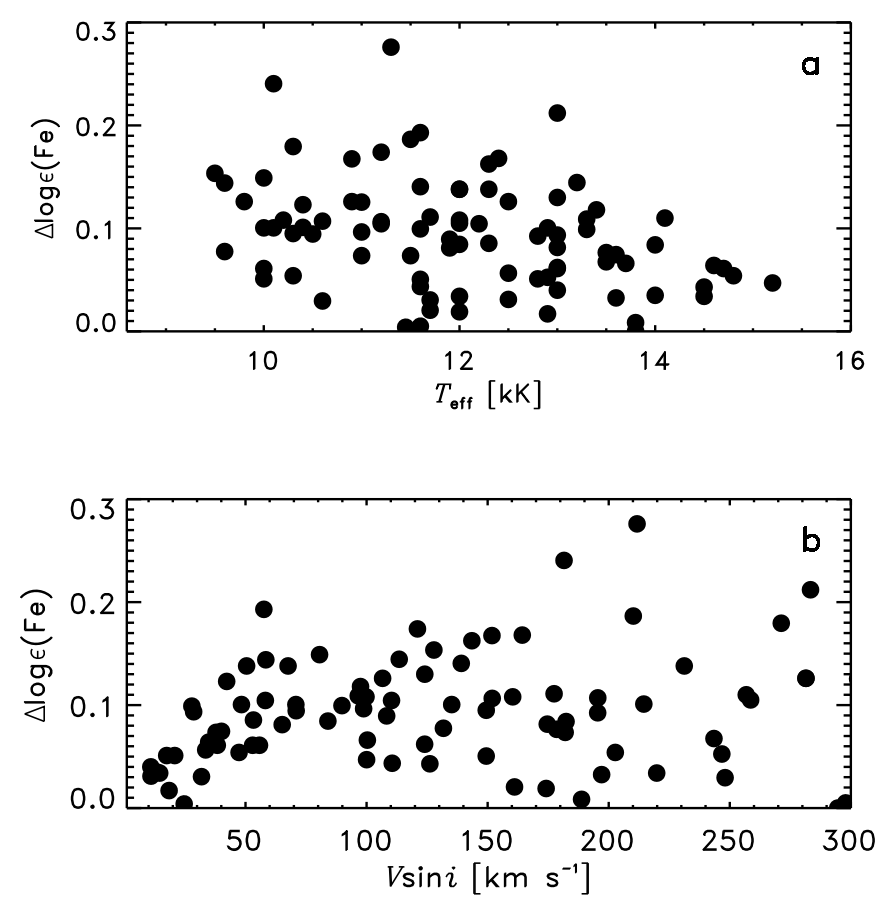

Fig. 7. Uncertainties in the $\mathrm{Fe}$ abundances caused by a change of $\xi$ by $1 \mathrm{~km} \mathrm{~s}^{-1}$, as a function of a) effective temperature and b) rotation velocity.

available in the stellar spectrum. There is a small correlation between $\Delta \log \varepsilon(\mathrm{Fe})$ and $V \sin i$ in the case of the change in the microturbulence (see Fig. 7). The scatter is larger for moderate and rapidly rotating stars. The impact of the uncertainties in the atmospheric parameters on our abundance results is discussed in more detail in Appendix A.
Table 3. Average abundances for the entire sample and the non-CP stars compared to the solar values (Grevesse et al. 2007).

\begin{tabular}{l|c|c|c}
\hline \hline El. & $\log \varepsilon(\mathrm{El})_{\text {all }}$ & $\log \varepsilon(\mathrm{El})_{\text {normal }}$ & Sun \\
\hline $\mathrm{He}$ & $10.94 \pm 0.18$ & $10.96 \pm 0.14$ & 10.93 \\
$\mathrm{C}$ & $8.21 \pm 0.41$ & $8.21 \pm 0.34$ & 8.39 \\
$\mathrm{~N}$ & $7.88 \pm 0.53$ & $7.64 \pm 0.17$ & 7.78 \\
$\mathrm{O}$ & $8.72 \pm 0.26$ & $8.74 \pm 0.26$ & 8.66 \\
$\mathrm{Ne}$ & $7.98 \pm 0.25$ & $8.01 \pm 0.26$ & 7.84 \\
$\mathrm{Mg}$ & $7.46 \pm 0.47$ & $7.56 \pm 0.37$ & 7.53 \\
$\mathrm{Al}$ & $6.02 \pm 0.85$ & $6.19 \pm 0.36$ & 6.37 \\
$\mathrm{Si}$ & $7.26 \pm 0.41$ & $7.22 \pm 0.31$ & 7.51 \\
$\mathrm{P}$ & $5.97 \pm 0.85$ & $5.63 \pm 0.37$ & 5.36 \\
$\mathrm{~S}$ & $7.06 \pm 0.52$ & $7.19 \pm 0.39$ & 7.14 \\
$\mathrm{Ca}$ & $6.33 \pm 0.61$ & $6.28 \pm 0.55$ & 6.31 \\
$\mathrm{Sc}$ & $3.12 \pm 0.37$ & $3.08 \pm 0.22$ & 3.05 \\
$\mathrm{Ti}$ & $5.03 \pm 0.54$ & $4.90 \pm 0.48$ & 4.90 \\
$\mathrm{~V}$ & $4.43 \pm 0.22$ & $4.38 \pm 0.23$ & 4.00 \\
$\mathrm{Cr}$ & $5.80 \pm 0.56$ & $5.59 \pm 0.34$ & 5.64 \\
$\mathrm{Mn}$ & $6.34 \pm 1.46$ & $5.17 \pm 0.59$ & 5.39 \\
$\mathrm{Fe}$ & $7.24 \pm 0.45$ & $7.13 \pm 0.29$ & 7.45 \\
$\mathrm{Ni}$ & $6.29 \pm 0.58$ & $6.23 \pm 0.62$ & 6.23 \\
$\mathrm{Sr}$ & $3.29 \pm 0.98$ & $2.63 \pm 0.36$ & 2.92 \\
$\mathrm{Y}$ & $2.90 \pm 0.18$ & $2.88 \pm 0.21$ & 2.21 \\
$\mathrm{Ba}$ & $2.76 \pm 0.52$ & $2.64 \pm 0.45$ & 2.17 \\
\hline
\end{tabular}

\section{Discussion of $V \sin i$}

All the projected rotational velocities obtained are listed in Table 2. We measured $V \sin i$ values ranging from 10 to about $300 \mathrm{~km} \mathrm{~s}^{-1}$. Low values of $V \sin i$ (up to $35 \mathrm{~km} \mathrm{~s}^{-1}$ ) were obtained for 13 stars. Moderate rotational velocities (from 40 to $80 \mathrm{~km} \mathrm{~s}^{-1}$ ) were derived for 20 objects. The sample with high $V \sin i$ values is the most populated and contains 56 stars, as can be seen in the histogram presented in Fig. 8. There is a small sample of very fast rotating stars, with $V \sin i$ values of about $200 \mathrm{~km} \mathrm{~s}^{-1}$ and more. The majority of stars have $V \sin i$ ranging from 80 to about $180 \mathrm{~km} \mathrm{~s}^{-1}$. The average $V \sin i$ value is $126 \mathrm{~km} \mathrm{~s}^{-1}$. Abt et al. (2002) investigated the rotational velocities of 1092 B-type stars by determining the line width of He I $\lambda 4471$ and $\mathrm{Mg}$ II $\lambda$ 44481. They found the average values for B6-B8 stars of luminosity class $\mathrm{V}$ and IV to be $152 \pm 8$ and $120 \pm 14 \mathrm{~km} \mathrm{~s}^{-1}$, respectively. For B9-B9.5 stars, the average values are $134 \pm 7$ and $99 \pm 14 \mathrm{~km} \mathrm{~s}^{-1}$ for V and IV luminosity classes, respectively. The average value obtained here for B6-B9.5 stars is consistent with the results of Abt et al. (2002). This is a qualitative comparison because we have a much smaller sample, but both samples are expected to be affected at the same level by selection biases (e.g., the under-representation of very fast rotators). As for the confirmed CPs, the candidate CPs appear to concentrate at much lower $V \sin i$ values than stars without any evidence of clear abundance peculiarities (Fig. 8). A K-S test shows that the probability that the $V \sin i$ values of the two samples of normal and CP stars are drawn from the same distribution is only about $7 \%$. This is consistent with the macroscopic motions arising from rotation impeding diffusion processes (see, e.g. Abt \& Morrell 1995 in the case of A stars). Furthermore, this supports the identification of our candidate CPs as bona fide members of this group.

\section{Results of abundance analysis}

The results of the abundance analysis are presented in Table 2. All abundances as given as $\log \varepsilon(\mathrm{El})$, where $\log \varepsilon(\mathrm{H})=12$ by convention. In Table 3, the average abundances of the stars in 


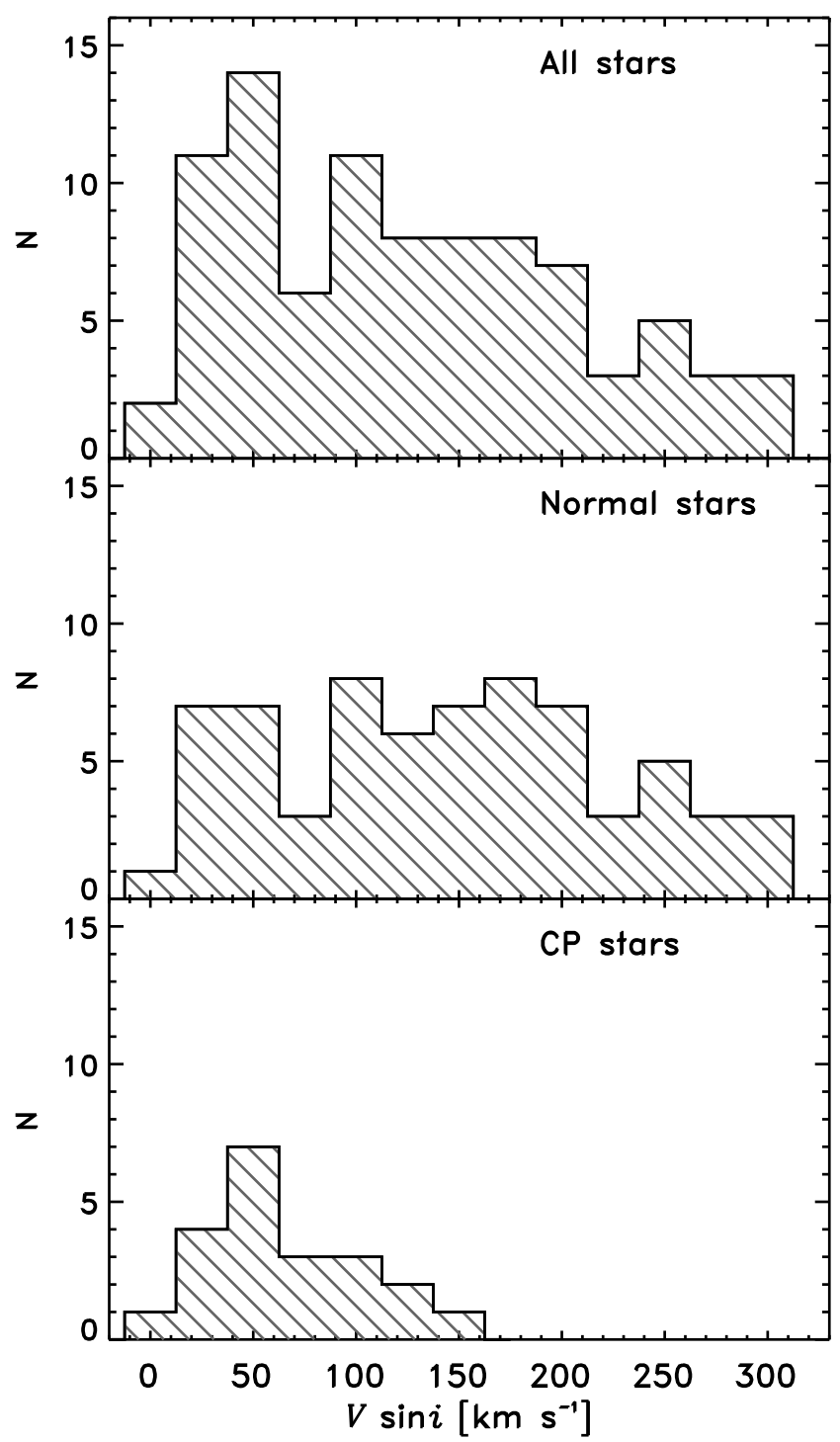

Fig. 8. The distribution of rotation velocities for all analysed stars (top panel), normal (non peculiar) objects (middle panel), and chemically peculiar stars (bottom panel). For the discussion of known and newly discovered chemically peculiar stars in our sample, see Sect. 7.

our sample are compared with the solar values (Grevesse et al. 2007). Because of the neglect of non-LTE effects, which can be large for some elements, significant caution should be exercised before considering the values for the non-CP objects as representative of the present-day abundances of the ISM in the solar neighbourhood as traced by late B-type stars.

For most of the stars, the chemical abundances were determined for the first time. For the stars for which information was already available in the literature, the references to the previous investigations are also given in Table 1. In Fig. 9, the determined chemical compositions for all stars are compared with the solar abundances of Grevesse et al. (2007). From this figure, we can see that lines of iron-peak elements are clearly evident in the spectra of all stars. However, only Fe was analysed for all of them. We also investigated $\mathrm{Sc}, \mathrm{Ti}, \mathrm{V}, \mathrm{Cr}, \mathrm{Mn}$, and $\mathrm{Ni}$ from this group. Lines of light elements are represented very sparsely in our spectra. We were able to determine the abundances of $\mathrm{He}, \mathrm{C}, \mathrm{N}, \mathrm{O}, \mathrm{Ne}, \mathrm{Mg}, \mathrm{Al}, \mathrm{Si}, \mathrm{P}, \mathrm{S}$, $\mathrm{Cl}$, and $\mathrm{Ca}$. We excluded the O I 7771-5 $\AA$ triplet from our analysis and relied instead on other O I lines (e.g., 5331, 6157,
6158 , and $6456 \AA$ ) that are much less affected by non-LTE effects (e.g., Przybilla et al. 2000). Most of the abundances of these elements were determined from one or two lines only. The determination of the helium abundance may be inappropriate if the $\mathrm{He}$ abundance remarkably differs from the solar value. In these cases, the continuum opacities require revision and new atmospheric models are required for consistency (e.g., Jeffery et al. $2001^{7}$ ). This may be the case for some stars in our sample. For example, $\mathrm{He}$ is significantly underabundant in HD 168932, HD 44948, HD 45153, HD 49935, HD 45583, HD 46340, HD 46886, HD 47756, HD 53851, HD 55362, and HD 56610. For all these stars, $\log \varepsilon(\mathrm{He})$ is lower than 0.40 . In a few cases, the He abundance is markedly higher than the solar value. This situation occurs for HD 179124, HD 44720 , HD 47431, HD 47759, HD 50252, and HD 56006. The heavy elements $(\mathrm{Ga}, \mathrm{Sr}, \mathrm{Y}, \mathrm{Zr}, \mathrm{Ba})$ were investigated only for slowly and moderately rotating stars. In most cases, only one line was available.

In Fig. 10, the histograms showing the distribution of the most common elements for the analysed late-B stars are presented. The majority of the stars have iron abundances lower than the solar value of 7.45 dex (Grevesse et al. 2007). However, there are some stars with enhanced iron abundances. In the extreme case of the chemically peculiar star HD 45583, Fe is overabundant by about 2 dex. We should point out that if the Fe abundance is very high, then the line blanketing will be stronger. Thus, for consistency, the atmospheric models should in principle be computed with opacity distribution functions corresponding to these higher abundances. The average Fe abundance is $7.24 \pm 0.45 \mathrm{dex}$ and $7.13 \pm 0.29 \mathrm{dex}$ for the entire sample and the non-CP stars, respectively. As discussed above, only nonLTE corrections at the 0.1 dex level are expected for Fe. The majority of the stars also have Si abundances lower than the solar value. This can be the result of a purely LTE analysis. As discussed by Hempel \& Holweger (2003), the non-LTE abundances of $\mathrm{Si}$ in this temperature range are higher than the LTE values. The non-LTE corrections amount to about $0.2 \mathrm{dex}$, while the average $\mathrm{Si}$ abundance determined here is lower than the solar value by about 0.25 dex. The iron-peak elements $\mathrm{Cr}$ and $\mathrm{Ti}$ have average abundances close to the solar ones. For three CP stars (HD 47759, HD 49713, and HD 45583), the $\mathrm{Cr}$ abundances are enhanced.

In Fig. 11, the iron abundances are shown as a function of $V \sin i$ and $T_{\text {eff }}$. There is no correlation between rotation velocity and $\log \varepsilon(\mathrm{Fe})$. On the other hand, the enhanced $\mathrm{Fe}$ abundances occur in most cases for stars with slow and moderate velocity. Similar dependencies were also investigated for all the other elements. A correlation between the abundances and the effective temperature was only found for $\mathrm{Mn}$ and $\mathrm{Ti}$. In both cases, the abundances are higher with increasing $T_{\text {eff }}$. These correlations can be produced because in normal, non-CP stars, the Ti and $\mathrm{Mn}$ lines are weaker at higher temperatures and become significantly blended with weak, unaccounted-for features of other elements (especially for rapid rotators). Therefore, we may overestimate $\log \varepsilon(\mathrm{Ti})$ and $\log \varepsilon(\mathrm{Mn})$ for such stars. In the case of $\mathrm{Mn}$, however, this correlation could have a physical cause (e.g. Jomaron et al. 1999).

\section{Chemically peculiar stars}

Among the upper main-sequence stars in the HR diagram, there exist a number of objects with unusual surface properties, e.g.,

7 http://star.arm.ac.uk/csj/models/Grid.html 


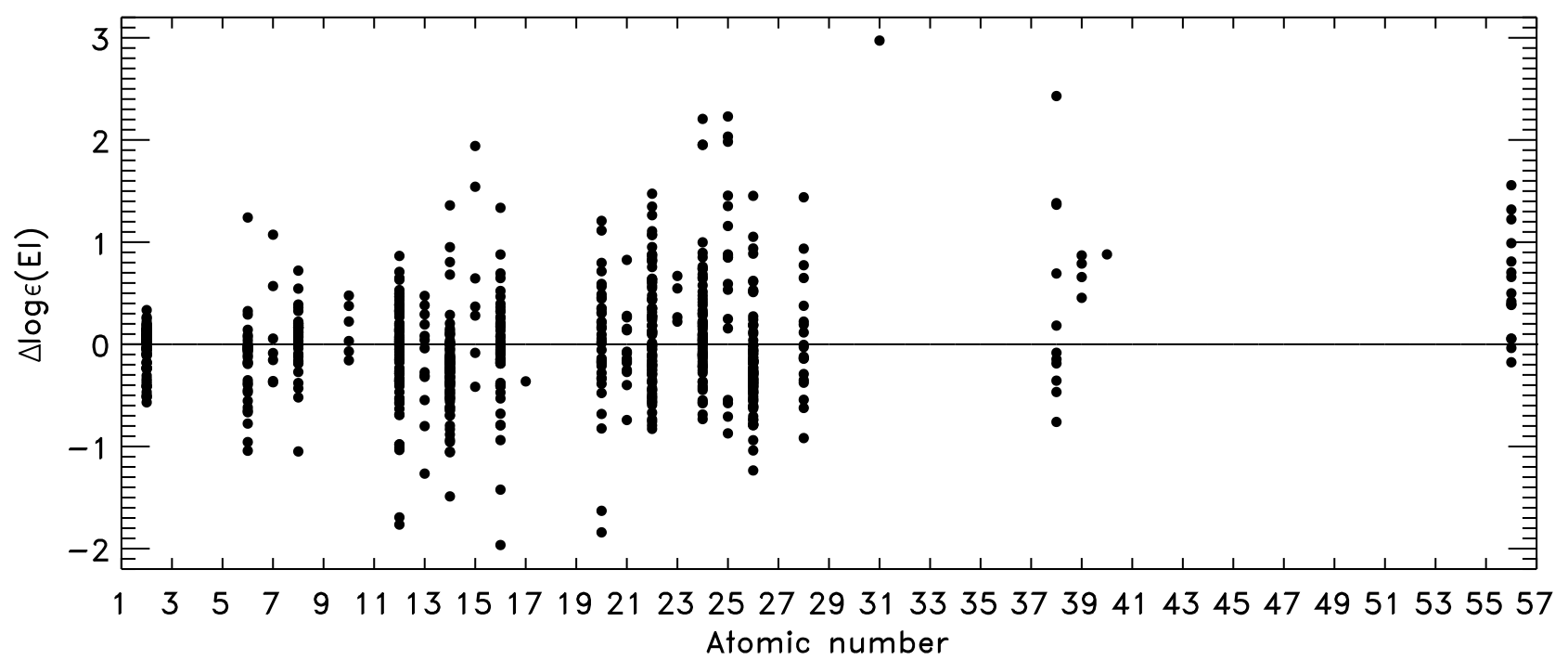

Fig. 9. Obtained chemical abundances of all analysed stars compared with the solar values (Grevesse et al. 2007). The differences between stellar and solar abundances, $\Delta \log \varepsilon(\mathrm{El})$, are shown as a function of the atomic number.
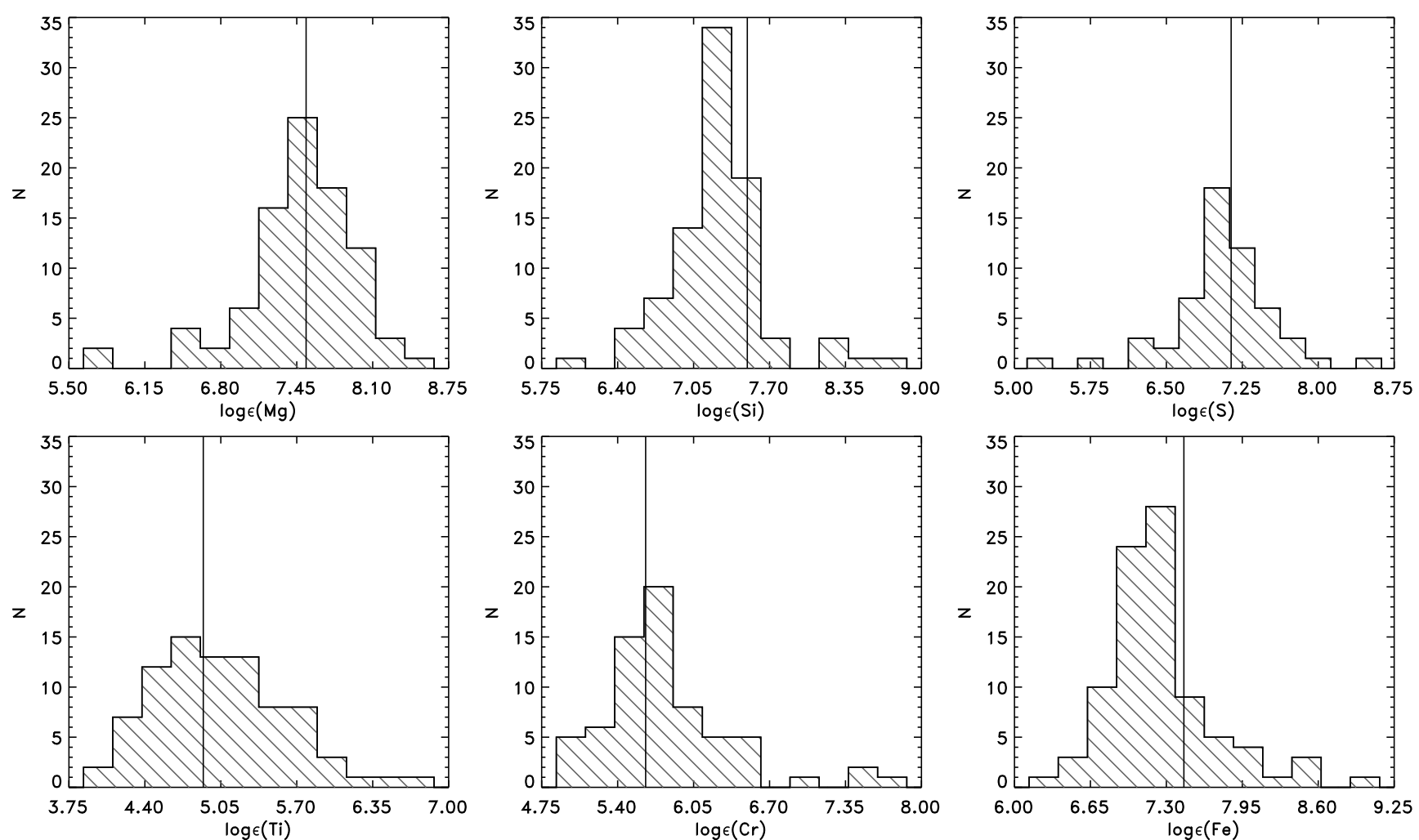

Fig. 10. The distributions of the abundances of the most common elements: $\mathrm{Mg}, \mathrm{Si}, \mathrm{S}, \mathrm{Ti}, \mathrm{Cr}$, and Fe. The solar abundances (Grevesse et al. 2007) are shown as straight lines.

peculiar abundances of some elements. The chemically peculiar B-type stars (CP, also called Bp stars) show a variety of abundance patterns known to be associated with strong magnetic fields. Classical Bp stars have extreme overabundances of rare-earth elements and significant overabundances of $\mathrm{Si}$. The latter is the most obvious anomaly in classical Bp stars. In the same range of temperatures, another group of peculiar stars exists. The mercury-manganese $(\mathrm{HgMn})$ stars constitute a clearly defined sub-group of CP stars with B spectral types. They are slowly rotating, non-magnetic (i.e., a magnetic field is not definitely detected for them) and mostly young stars. The chemical peculiarities are the effect of microscopic diffusion. These stars exhibit abundance anomalies of several elements, e.g., overabundances of $\mathrm{Hg}, \mathrm{Mn}, \mathrm{Y}, \mathrm{P}, \mathrm{Sr}$, and $\mathrm{Zr}$, and deficiencies in $\mathrm{He}, \mathrm{Al}$, $\mathrm{Fe}, \mathrm{Zn}, \mathrm{Ni}$. Additionally, the $\lambda$ Bootis stars are non-magnetic, Population I dwarfs, with spectral types from late-B to early F. 

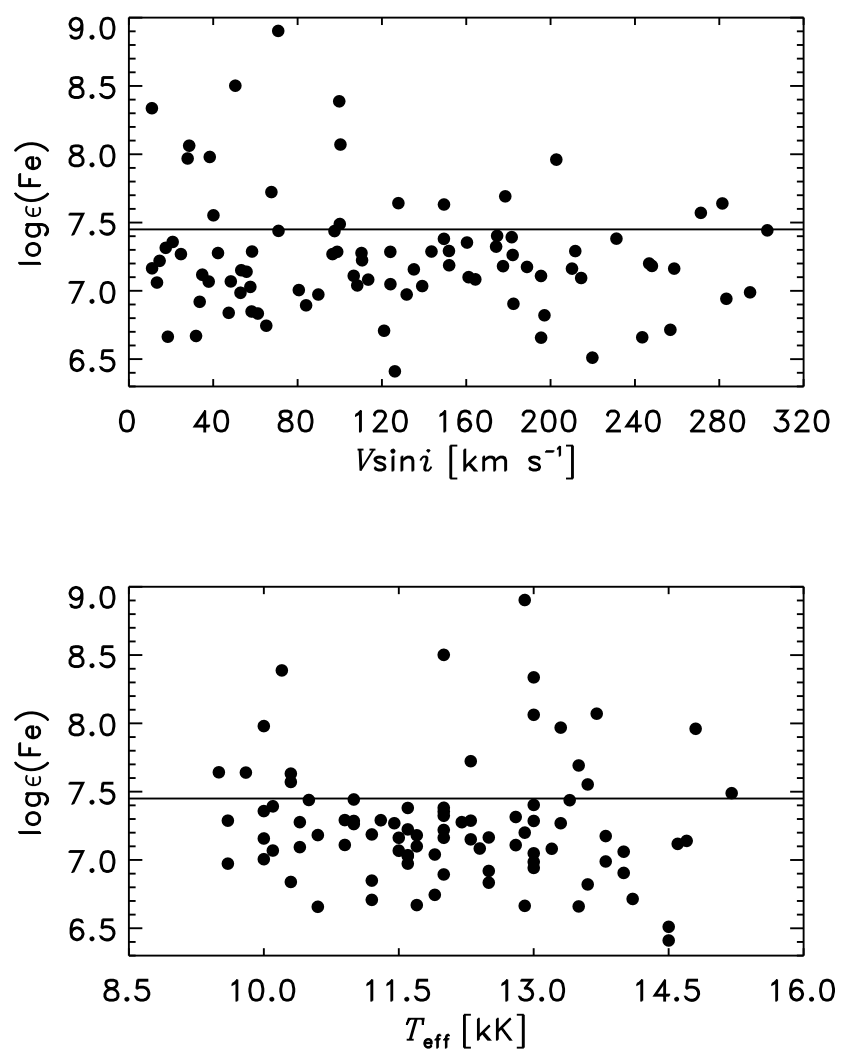

Fig. 11. The obtained stellar Fe abundances, as a function of rotational velocity $V \sin i$ (top panel) and effective temperature (bottom panel). The horizontal line is the solar value (Grevesse et al. 2007).

They show significant underabundances of metals, whereas the light elements $(\mathrm{C}, \mathrm{N}, \mathrm{O}$, and $\mathrm{S})$ have almost normal abundances compared to the Sun.

Our abundance determination of 89 late B-type stars in the CoRoT field allowed us to discover 9 new suspected CP stars and to determine for the first time abundances for 7 stars previously classified as chemically peculiar. The abundances of these objects are compared with the solar values in Figs. 12 and 13.

Below, we provide a star-by-star description of the properties of each of those objects.

\subsection{New chemically peculiar stars}

HD 45975, NGC 2232-6, Sp. type B9, V sin $i=61 \pm 3 \mathrm{~km} \mathrm{~s}^{-1}$. Jenkner \& Maitzen (1987) searched for CP stars in the NGC 2232 cluster. According to them, this star appears to be normal. However, they were looking only for CP2 stars (the classical magnetic peculiar stars), so they may have easily missed some other type of peculiarity. We determined a moderate rotational velocity for this star. We derived solar or close to solar abundances for all analysed light elements. On the other hand, all considered iron-peak elements besides iron are overabundant. The most enhanced abundances were obtained for Mn. This abundance pattern shows some similarities with $\mathrm{HgMn}$ stars, so HD 45975 can be suspected to be another member of this class.

HD 46886, Sp. type B8, V sin $i=18 \pm 1 \mathrm{~km} \mathrm{~s}^{-1}$. We determined a low rotational velocity and we were able to analyse the abundances of 19 elements. The abundances of all light elements, apart from $\mathrm{Ne}, \mathrm{P}$, and $\mathrm{Ca}$, are underabundant compared to the solar values. All iron-peak elements besides $\mathrm{Fe}$ and $\mathrm{Ni}$ are enhanced. The iron abundance is lower than the solar value.

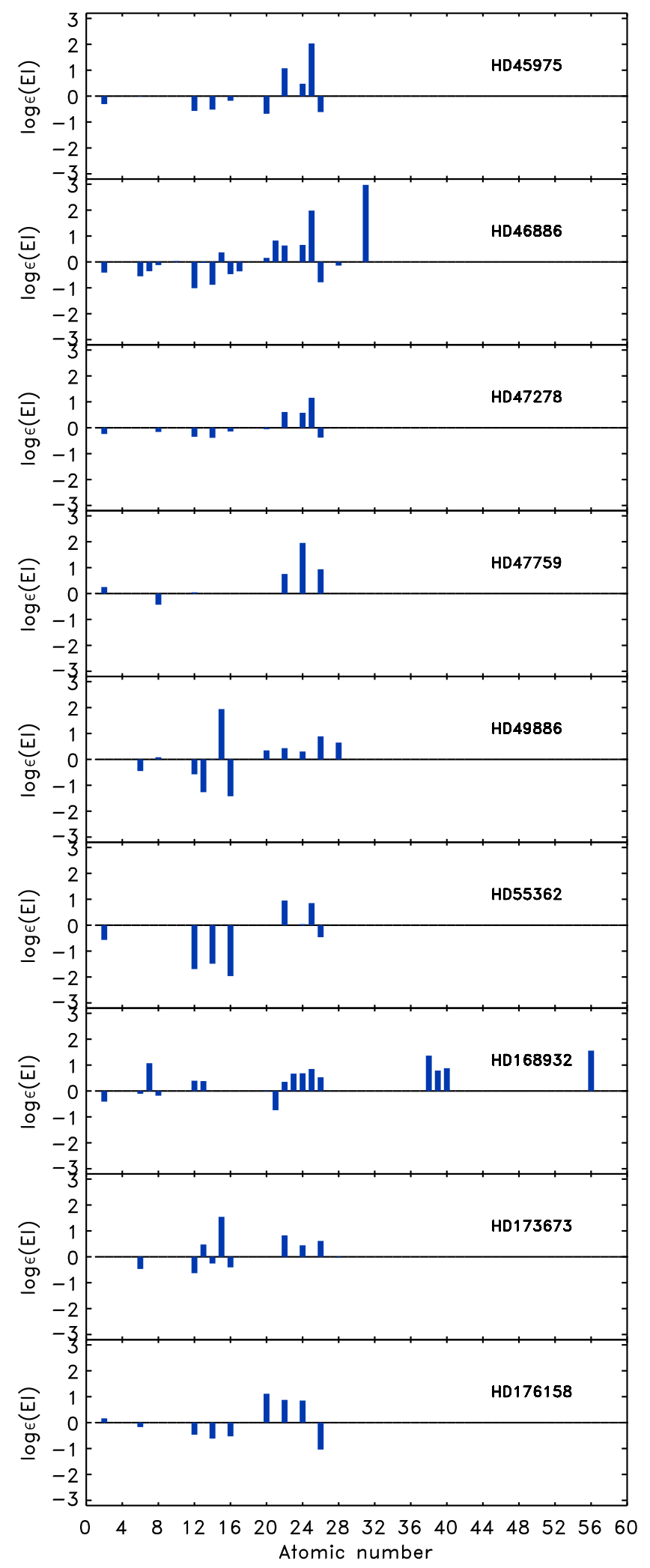

Fig. 12. Elemental abundances of the new suspected chemically peculiar stars compared with the solar abundances (Grevesse et al. 2007).

The abundance of Mn is enhanced by more than 2 dex. The only analysed heavy element, $\mathrm{Ga}$, is overabundant by about 3 dex. On the basis of this abundance pattern, we can classify HD 46886 as a HgMn star. 


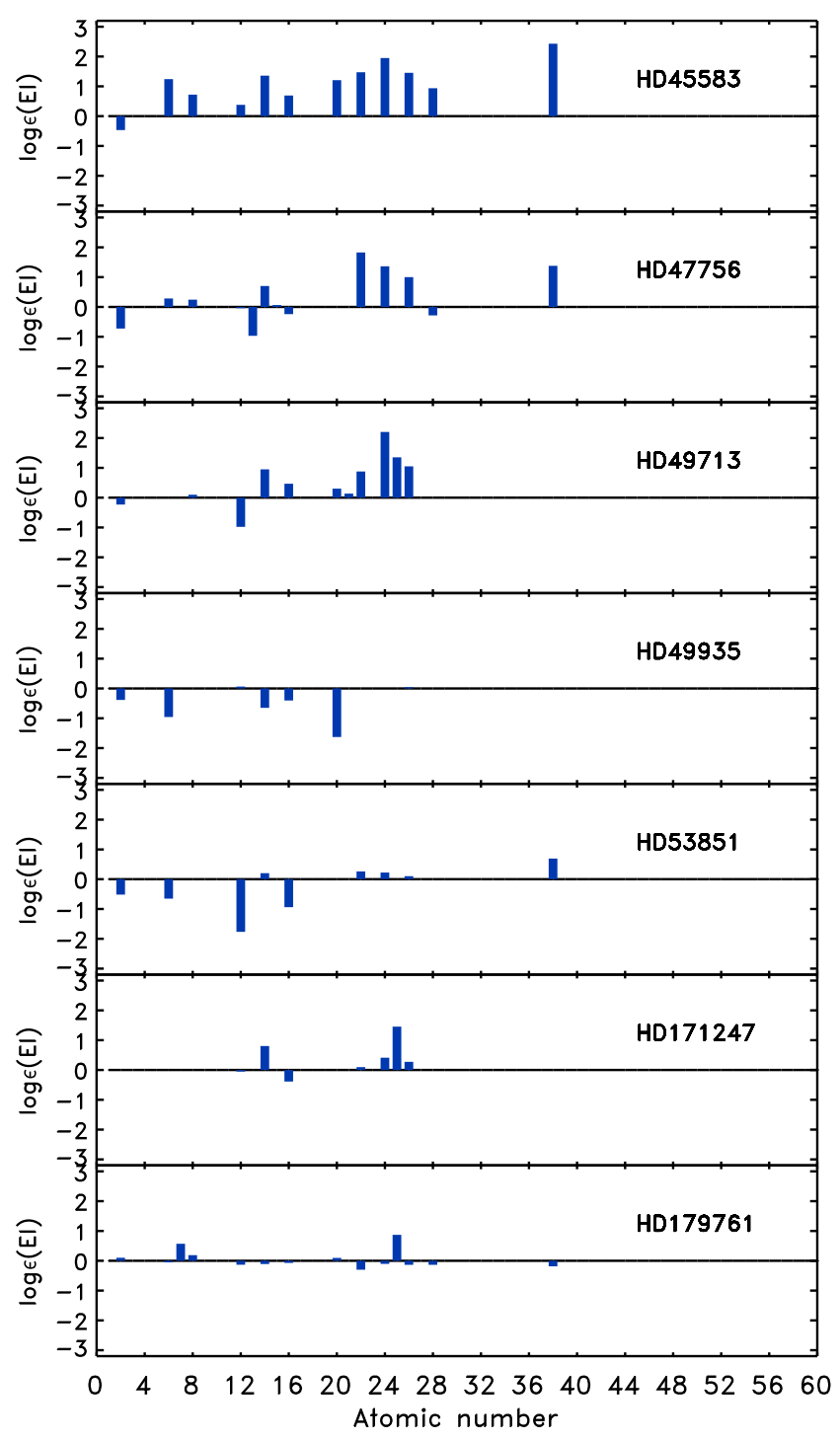

Fig. 13. Elemental abundances of known chemically peculiar stars compared with the solar abundances (Grevesse et al. 2007).

HD 47278, Sp. type B9, V $\sin i=38 \pm 2 \mathrm{~km} \mathrm{~s}^{-1}$. We derived a moderate rotational velocity. All light elements investigated are underabundant. All analysed iron-peak elements besides Fe are overabundant. The abundance of $\mathrm{Mn}$, obtained from a single line, is enhanced by about 1 dex. Because of the small number of lines available for the analysis, we cannot conclude anything about the type of peculiarity of this object, but it could be related to $\mathrm{HgMn}$ stars.

HD 47759, V 753 Mon, Sp. type B9, V $\sin i=100 \pm 4 \mathrm{~km} \mathrm{~s}^{-1}$. HD 47759 was classified as a variable star of the $\alpha^{2}$ CVn-type by Kazarovets (1999). There is no information about the chemical peculiarity of this object, but $\alpha^{2} \mathrm{CVn}$ stars are rotating variables of spectral type B8p to A7p with strong magnetic fields. We found a high rotational velocity. The only light elements that we were able to analyse for this rotation velocity were $\mathrm{He}, \mathrm{O}$, and $\mathrm{Mg}$. For all of them, only a single line is measurable in the spectrum. The iron-peak elements considered were $\mathrm{Ti}, \mathrm{Cr}$, and Fe. All iron-peak elements are overabundant by about 1 to 2 dex. All elemental abundances except those for Fe were derived from 1 or 2 spectral features.
HD 49886, Sp. type B8, V $\sin i=11 \pm 1 \mathrm{~km} \mathrm{~s}^{-1}$. The obtained rotational velocity is very low and allows a detailed abundance analysis to be completed. The majority of the analysed light elements are underabundant. Only $\mathrm{O}$ and $\mathrm{P}$ have enhanced abundances. The iron-peak elements have abundances close to solar values or are overabundant. The most reliable abundances were determined for iron-peak elements and two light elements, $\mathrm{Si}$ and $\mathrm{P}$, for which more than 3 lines were analysed.

HD 55362, Sp. type B9, V $\sin i=53 \pm 6 \mathrm{~km} \mathrm{~s}^{-1}$. The abundance pattern of this star is similar to HD 47278, and may indicate that it is another $\mathrm{HgMn}$ star. The abundances of the light elements are significantly lower than the solar ones. All ironpeak elements considered, apart from Fe are overabundant. The abundance of $\mathrm{Mn}$ is enhanced by about 1 dex.

HD 168932, Sp. type B9, $V \sin i=38 \pm 1 \mathrm{~km} \mathrm{~s}^{-1}$. There is no determination of atmospheric parameters and chemical abundances in the literature for this star, but it was considered to be a $\lambda$ Bootis candidate by Paunzen et al. (2001). We determine a moderate rotational velocity. The abundances of the light elements differ only slightly from solar values. The most overabundant element in this group is N. All analysed iron-peak elements besides Sc are overabundant. All heavy elements are significantly overabundant, but the analysis was performed for 1 or 2 lines of each element only. This result is inconsistent with the characteristics of a typical $\lambda$ Bootis star, but the abundance pattern of this star is peculiar.

HD 173673, Sp. type B8, V sin $i=29 \pm 2 \mathrm{~km} \mathrm{~s}^{-1}$. The moderate rotation allowed us to determine the abundances of 10 elements. The light elements turn out to be underabundant except $\mathrm{Al}$, which appeared to be solar, and $\mathrm{P}$, which is significantly overabundant. All analysed iron-peak elements are overabundant. Only for $\mathrm{Fe}, \mathrm{Ti}, \mathrm{Si}, \mathrm{Cr}$, and $\mathrm{S}$ are there more than 2 lines available in the spectra. The results obtained for these elements are the most reliable. The other abundances were determined from 1 or 2 features only. Considering the overabundance of $\mathrm{P}$, and the deficiency in Al, we can conclude that HD 173673 is a candidate $\mathrm{HgMn}$ star.

HD 176158, Sp. type B9, V $\sin i=126 \pm 5 \mathrm{~km} \mathrm{~s}^{-1}$. We derived a high rotational velocity for this star. The abundances of all light elements apart from He are lower than the solar values. All investigated iron-peak elements besides $\mathrm{Fe}$ are overabundant. The differences with the solar abundances can be about 1 dex. Only 1 or 2 lines were available for all elements besides He.

The abundance patterns and rotational velocities of the stars listed above indicate that these objects can be chemically peculiar. We also discovered other stars with peculiarities in their spectra, but with high rotation velocities or noisy spectra. The results for them are therefore less reliable, as indicated in the following:

HD 46340, Sp. type B8, $V \sin i=128 \pm 4 \mathrm{~km} \mathrm{~s}^{-1}$. We found that all elements apart from $\mathrm{He}$ and $\mathrm{Sr}$ have slightly enhanced abundances. This could be caused by binarity, as in The Hipparcos and Tycho Catalogues (Perryman 1997) this star is defined to be binary with a separation between the components $\rho=0.650^{\prime \prime}$. The same separation is given in the Tycho Double Star Catalogue (Fabricius et al. 2002). In the All-sky Compiled Catalogue of 2.5 million stars, Kharchenko (2001) quotes the $V$ magnitudes of both components to be 7.64 and 8.39 mag, respectively.

HD 51079, Sp. type B8 V, V sin $i=161 \pm 8 \mathrm{~km} \mathrm{~s}^{-1}$. We measured a high rotation velocity for this object. For this rotation value, only as many as 3 lines were available for all elements. The abundances of all elements apart from $\mathrm{Cr}$ are lower or close to the solar values. Chromium is overabundant by about $1 \mathrm{dex}$. 
HD 53004, Sp. type B9, V $\sin i=58 \pm 8 \mathrm{~km} \mathrm{~s}^{-1}$. We obtained a moderate rotation velocity. All investigated light elements are underabundant. All iron-peak elements apart from Mn also have lower abundances than solar. Manganese is overabundant by more than 1 dex. We classified this star as a possible $\mathrm{HgMn}$ object.

HD 56610, Sp. type B9, V $\sin i=100 \pm 10 \mathrm{~km} \mathrm{~s}^{-1}$. We obtained a high rotational velocity. The investigated light elements are underabundant. On the other hand, all considered iron-peak elements have enhanced abundances.

HD 168202, Sp. type B9, V $\sin i=71 \pm 6 \mathrm{~km} \mathrm{~s}^{-1}$. All light elements apart from $\mathrm{Ca}$ and $\mathrm{Mg}$ are underabundant. Among the iron-peak elements, only $\mathrm{Mn}$ is overabundant. The abundance of the rare-earth element $\mathrm{Ba}$ is also enhanced. Only the abundances of $\mathrm{Ti}, \mathrm{Cr}$, and $\mathrm{Fe}$ were derived from the analysis of more than 3 lines. If the results are reliable, HD 168202 can be classified as a suspected HgMn star.

\subsection{Known chemically peculiar stars}

HD 45583, V682 Mon, Sp. type B8 Si, V sin $i=71 \pm 6 \mathrm{~km} \mathrm{~s}^{-1}$. This star is a member of NGC 2232, and was found to be peculiar by Levato \& Malaroda (1974) on the basis of spectroscopic observations. They discovered silicon lines visible at 4200, 3856, 3862, 4128, and $4130 \AA$ A. Levato \& Malaroda (1974) also found Cr II, Fe II, and Sr II abundances to be enhanced and a great number of other faint lines present in the spectrum. Bagnulo et al. (2006) and Kudryavtsev et al. (2006) independently discovered a magnetic field in this star. HD 45583 is classified as a variable of the $\alpha^{2} \mathrm{CVn}$ type. Two possible periods were discovered by North (1987). In the catalogue of Catalano \& Renson (1998), a period of $1.177 \mathrm{~d}$ is given. Effective temperatures of 12600 and $13200 \mathrm{~K}$ were determined by Glagolevskij (1994) from Q and $\mathrm{X}$ indices, respectively. The results of a detailed study of HD 45583 were reported by Semenko et al. (2008), who found a period of spectral and magnetic variability coincident with the rotation period of $1.177 \mathrm{~d}$. These authors determined $T_{\text {eff }}=$ $13000 \mathrm{~K}$ and $\log g=4.0$. According to Semenko et al. (2008), some elements ( $\mathrm{Fe}, \mathrm{Si}$, and $\mathrm{Cr}$ ) show a 1-2 dex overabundance and $\mathrm{He}$ is underabundant by about 2 dex with respect to the Sun. We obtained a moderate rotational velocity and we analysed the lines of 12 elements. All light elements apart from He are overabundant. All iron-peak and heavy elements are overabundant. The most enhanced abundances were derived for $\mathrm{Cr}, \mathrm{Fe}$, and the heavy element $\mathrm{Sr}$. These results are consistent with the previous ones in the literature.

HD 47756, Sp. type: B8 SiCrSr, V sin $i=27 \pm 1 \mathrm{~km} \mathrm{~s}^{-1}$. This star is a member of the NGC 2244 cluster. Cowley \& Crawford (1971) found HD 47756 to be peculiar on the basis of photometric criteria. Glagolevskij (1994) obtained $T_{\text {eff }}=12800$ and $12400 \mathrm{~K}$ from Q and X indices, respectively. Kudryavtsev et al. (2006) discovered a magnetic field. We derive a low rotation velocity. Our study is the first to determine the abundance pattern of this star. All investigated light elements apart from $\mathrm{Si}$ are slightly underabundant. The $\mathrm{Si}$ abundance is close to the solar value. All the iron-peak element except Ni are overabundant. The most enhanced element is Ti. The heavy element $\mathrm{Sr}$ is also overabundant by about $1.5 \mathrm{dex}$, but this value was determined for one line only. The chemical composition is typical of B8 SiSrCr stars.

HD 49713, V740 Mon, Sp. type B9p (CrEuSi), $V \sin i=50 \pm$ $2 \mathrm{~km} \mathrm{~s}^{-1}$. HD 49713 was classified as peculiar by Walther (1949) on the basis of spectroscopic observations and was classified as a variable chemically peculiar star of the $\alpha^{2}$ CVn type by Kazarovets (1999). Kudryavtsev et al. (2006) discovered a magnetic field. We present the first determination of the atmospheric parameters and detailed chemical composition of this star. We derived a moderate rotational velocity which allows us to study the abundances of 11 elements. All investigated light elements, apart from $\mathrm{Mg}$ have abundances close to the solar values. All iron-peak elements are overabundant, with the exception of Sc. The most overabundant is $\mathrm{Cr}$. Only the abundances of $\mathrm{Si}, \mathrm{Ti}, \mathrm{Cr}$, and Fe were obtained on the basis of more than 3 lines. The derived chemical pattern is typical of Bp stars.

HD 49935, V741 Mon, Sp. type B8, V sin $i=100 \pm 14 \mathrm{~km} \mathrm{~s}^{-1}$. Kazarovets (1999) classified this star as a variable star of the $\alpha^{2}$ CVn type. All analysed light elements, including Si are underabundant. We were able to analyse only Fe of iron-peak elements. The iron abundance is close to the solar value. Only the Fe abundance was determined from more than 3 features. On the basis of these results, we cannot classify this star as chemically peculiar.

HD 53851, Sp. type B9, $V \sin i=40 \pm 3 \mathrm{~km} \mathrm{~s}^{-1}$. This star was classified as chemically peculiar by Vogt \& Faundez (1979) from the analysis of Strömgren photometry. Koen \& Eyer (2002) found HD 53851 to be variable with a period of $0.49396 \mathrm{~d}$. Beside of this, there is little information about HD 53851 in the literature. We derived a moderate rotation velocity. All light elements apart from Si are underabundant. All analysed iron-peak elements and $\mathrm{Sr}$ are slightly overabundant. We classify this object as a B9SiCrSr star.

HD 171247, V2393 Oph, Sp. type B8IIIsp, B8Si, V $\sin i=$ $68 \pm 2 \mathrm{~km} \mathrm{~s}^{-1}$. This star was classified as peculiar by Whitford (1963) on the basis of spectroscopic observations. Glagolevskij (1994) determined the effective temperatures to be 12000 and $11200 \mathrm{~K}$ from Q and X photometry, respectively. North \& Kroll (1989) derived an effective temperature of 11910 K. V 2393 Oph was claimed to be a variable star of the $\alpha^{2} \mathrm{CVn}$ type. Leone et al. (1995) discussed high-resolution spectroscopic observations and non-LTE calculations of the He I $10830 \AA$ line in magnetic chemically peculiar stars, including HD 171247. They determined $V \sin i=60 \mathrm{~km} \mathrm{~s}^{-1}$. Leone \& Manfre (1996) performed a spectroscopic study of HD 171247 and adopted $T_{\text {eff }}=12166 \mathrm{~K}$ and $\log g=3.42$. They derived the abundances with WIDTH9. The microturbulent velocity, $\xi=2.1 \mathrm{~km} \mathrm{~s}^{-1}$, was inferred from unblended $\mathrm{Fe}$ lines. All unblended lines have been used to determine the rotational velocity, $V \sin i=50 \mathrm{~km} \mathrm{~s}^{-1}$. Leone \& Manfre (1996) determined the abundances of $\mathrm{Si}, \mathrm{Cr}$, and $\mathrm{Fe}$ (all overabundant by $0.8 \mathrm{dex}$ ). The photometric variability of this star was discovered by North (1992), who found the period to be 3.9124 d, as confirmed by Catalano \& Renson (1998). Kazarovets et al. (1999) included this star in the list of variables of the $\alpha^{2}$ CVn type. Catanzaro et al. (1999) detected variability in He I $5876 \AA$ A. They adopted $T_{\text {eff }}=11300 \mathrm{~K}$ and $\log g=$ 3.4 from Strömgren photometry. All elements investigated here have abundances close to the solar values or are overabundant.

HD 179761, V 1288Aql, 21 Aql, Sp. type B8II-III, $V \sin i=$ $17 \pm 1 \mathrm{~km} \mathrm{~s}^{-1}$. This star has frequently been investigated in the past. The first determination of chemical abundances was performed by Searle et al. (1966, see references therein), Sargent et al. (1969), and Durrant (1970). Bertaud (1959) classified V 1288 Aql as a B8 Si star. Kukarkin (1972) placed this star in his list of variables. Adelman (1984), Adelman \& Fuhr (1985), and Adelman (1991) performed the analysis of lines in the optical region and derived abundances close to the solar ones. Roby \& Lambert (1990) obtained C, N, and O abundances for 
chemically peculiar stars, including 21 Aql. They found $\mathrm{C}$ and $\mathrm{O}$ abundances to be in general agreement with radiative diffusion theory. Adelman et al. (1993) performed the analysis of iron-peak elements on the basis of IUE ultraviolet spectra and found good agreement between UV and optical determinations. From IUE observations, Smith (1993) and Smith \& Dworetsky (1993) also obtained chemical abundances of light and iron-peak elements. They derived approximately solar abundances of Ti, Cr, Mn, Fe, and Ni. Smith (1994, 1996, 1997) continued the analysis of coadded IUE spectra and investigated the ultraviolet lines of $\mathrm{Co}, \mathrm{Zn}, \mathrm{Ga}$, and $\mathrm{Hg}$. It turned out that $\mathrm{Hg}$ is slightly overabundant in this star. Catalano \& Renson (1998) placed $\mathrm{V} 1288 \mathrm{Aql}$ in their list of variables as a probable $\mathrm{B} 8 \mathrm{Hg}$ star. Adelman et al. (2004) analysed the lines of very heavy elements (Pt, $\mathrm{Au}, \mathrm{Hg}, \mathrm{Tl}$, and $\mathrm{Bi}$ ) in the UV spectra of $21 \mathrm{Aql}$. Bychkov et al. (2003) classified this star as a chemically peculiar silicon star. Ryabchikova (2005) considered the temperature behaviour of elemental abundances in the atmosphere of magnetic peculiar stars, including 21 Aql. For this star, Huang \& Gies (2006) derived $\log g=3.47, T_{\text {eff }}=12746 \mathrm{~K}$, and a projected rotational velocity of $12 \pm 6 \mathrm{~km} \mathrm{~s}^{-1}$. Cenarro et al. (2007) analysed medium-resolution spectra and adopted the stellar atmospheric parameters $T_{\text {eff }}=13175 \mathrm{~K}, \log g=3.27$, and $[\mathrm{Fe} / \mathrm{H}]=-0.14$ dex. The abundances derived here are in accordance with the previous determinations. All elements appear to be solar or slightly underabundant. Only $\mathrm{Mn}$ is overabundant by about 0.5 dex, but this is based on a single line. If the Mn abundance is reliable and taking into account the results of Smith (1997), we can classify $21 \mathrm{Aql}$ as a mild HgMn star.

\section{Conclusions}

We have analysed a sample of 89 late B-type stars of spectral types B6-B9.5 located in the CoRoT field of view. All the high-resolution spectra were obtained with the FEROS and/or ELODIE spectrographs and collected in the GAUDI database, although a renormalisation of all spectra turned out to be necessary. This research is part of a global programme for deriving the elemental abundances of all B-type stars in the CoRoT field with visual magnitude below $8.0 \mathrm{mag}$ and with spectra available in the GAUDI archive. In the next paper of this series, we will present the non-LTE abundance analysis of the hot B0-B5 stars (Niemczura et al., in preparation; Paper III).

In view of the large number of stars to be analysed, we made several basic assumptions in our study. First of all, we determined the effective temperatures of the stars from photometric calibrations and kept these fixed. Secondly, we fixed the microturbulence to a value of $2 \mathrm{~km} \mathrm{~s}^{-1}$ (or 0 and $1 \mathrm{~km} \mathrm{~s}^{-1}$ for a few stars). Thirdly, we did not allow any additional line-broadening to make the line-profile fits, i.e., we did not consider macroturbulence. Based on these assumptions, the surface gravities were derived from the comparison of observed and theoretical Balmer line profiles. For the adopted $T_{\text {eff }}, \log g$, and $\xi$ values, we were able to determine the chemical composition even for very rapidly rotating stars. The present study allowed us to derive the abundances of a large number of chemical species, including heavy and rare-earth elements. The dominant species in the spectrum of every star are iron-peak elements. The average Fe abundance for the entire sample is $7.24 \pm 0.45 \mathrm{dex}$, but there are stars for which $\log \varepsilon(\mathrm{Fe})$ significantly exceeds the solar value.

As a side result, we report the discovery of at least 9 new chemically peculiar stars, including Bp and HgMn stars. Two of the most interesting objects among them are HD 46886 and HD 45975, which are new HgMn stars with a very rich spectrum.
Table A.1. Stellar parameters and rotational velocites of the test synthetic spectra.

\begin{tabular}{c|c|c|c}
\hline \hline $\begin{array}{c}T_{\text {eff }} \\
{[\mathrm{K}]}\end{array}$ & $\log g$ & $\begin{array}{c}\xi \\
{\left[\mathrm{km} \mathrm{s}^{-1}\right]}\end{array}$ & $\begin{array}{c}V \sin i \\
{\left[\mathrm{~km} \mathrm{~s}^{-1}\right]}\end{array}$ \\
\hline 10000 & 4.0 & 2 & $10,50,100,150,200$ \\
12000 & 4.0 & 2 & $10,50,100,150,200$ \\
14000 & 4.0 & 2 & $10,50,100,150,200$ \\
\hline
\end{tabular}

All the other non-peculiar stars have abundance patterns that are consistent with typical abundances of B-type stars.

Our determination of the fundamental parameters and abundance patterns will serve as an important input to future seismic modelling of the stars based on CoRoT data.

Acknowledgements. The authors wish to thank the referee, Douglas Gies, for useful comments. This study was funded by means of a 6-month Visiting Postdoctoral Fellowship assigned to EN by the Fund for Scientific Research of Flanders (FWO) in the framework of FWO project G.0332.06. The authors are supported by the Research Council of Leuven University under grant GOA/2008/04 and by the European Helio- and Asteroseismology Network (HELAS), a major international collaboration funded by the European Commission's Sixth Framework Programme.

\section{Appendix A: Reliability of the method and effect of the uncertainties in the atmospheric parameters on the abundance results}

To assess the robustness of the procedures used to determine the chemical abundances, we computed a grid of synthetic spectra and compared the input values with the results derived by our method. The choice of parameters for the grid was dictated by the range in effective temperatures of our sample. Typical surface gravity and microturbulence were adopted: $\log g=4.0$ dex and $\xi=2 \mathrm{~km} \mathrm{~s}^{-1}$. We broadened the spectra with five different rotational velocities sampling the range covered by our stars (10-200 $\mathrm{km} \mathrm{s}^{-1}$ ) to check whether our method recovers the true abundances even for rapid rotators. The parameters adopted for the grid of theoretical spectra are presented in Table A.1. Solar metallicity was assumed, but the individual abundances of some elements were slightly changed.

All the synthetic spectra were degraded by white, Gaussian noise to achieve a signal-to-noise ratio of about 100 typical of our data (the synthetic spectra also have a resolving power of 40000 matching that of the observed spectra).

In Fig. A.1, we compare the obtained chemical abundances $\left(\log \varepsilon(\mathrm{El})_{\text {obtained }}\right)$ with the input values adopted for the synthetic spectra $\left(\log \varepsilon(\mathrm{El})_{\text {model }}\right)$. The differences are plotted with different symbols depending on the assumed rotational velocity. For clarity, the results for a given temperature are presented in separate panels. The discrepancies are in most cases lower than $0.1 \mathrm{dex}$. For $T_{\text {eff }}=10000 \mathrm{~K}$, the largest differences are found for $V \sin i=$ $200 \mathrm{~km} \mathrm{~s}^{-1}$, while for $T_{\text {eff }}=12000 \mathrm{~K}$ they are lower than 0.1 in all cases. For $T_{\text {eff }}=14000 \mathrm{~K}$, the largest discrepancies are found for $V \sin i=150 \mathrm{~km} \mathrm{~s}^{-1}$ in the case of $\mathrm{Ca}$ and $\mathrm{Ni}$. The lines are very weak in this case, and for $V \sin i=200 \mathrm{~km} \mathrm{~s}^{-1}$, they are no longer measurable.

This test demonstrates the usefulness of our method for the determination of the abundance pattern of late B-type stars even at high rotation velocities. Only in the case of weak, blended features are the results prone to large uncertainties. 


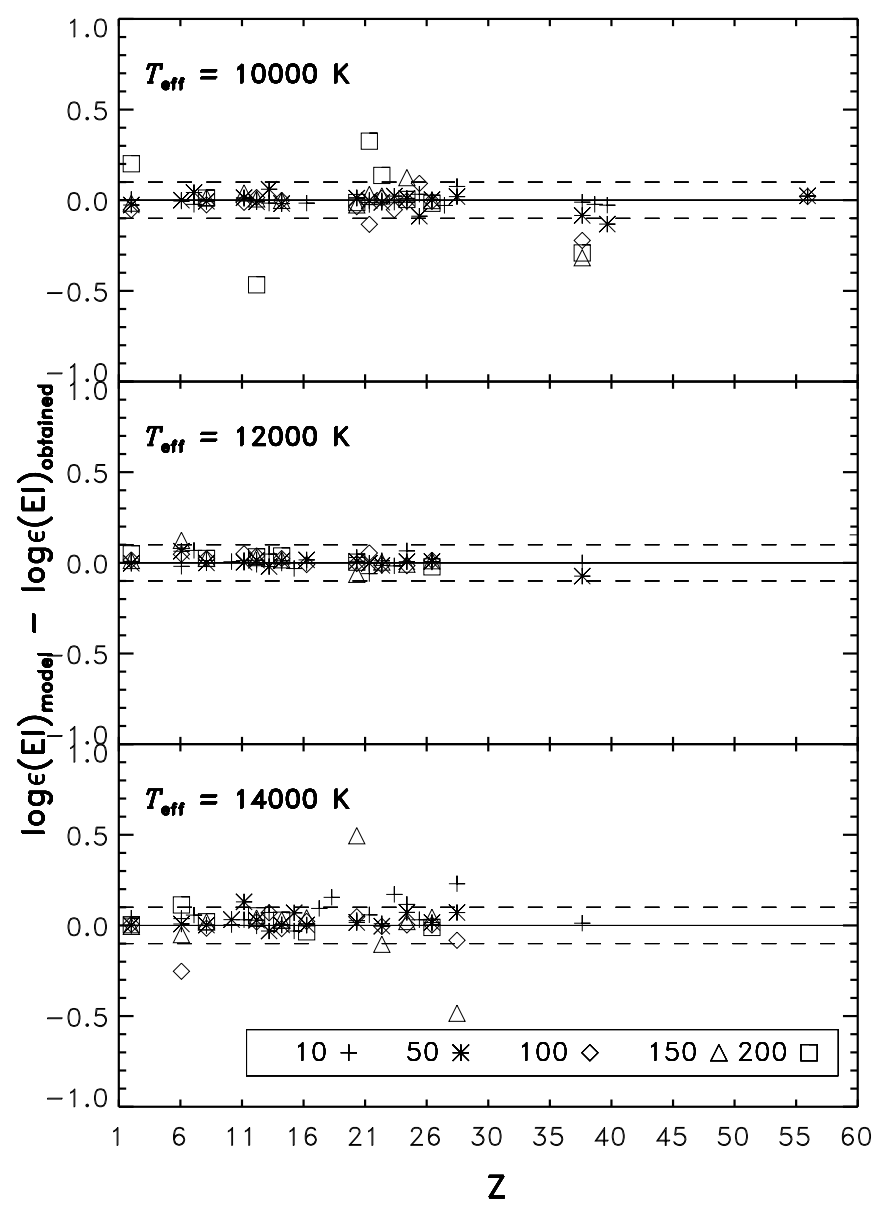

Fig. A.1. Differences between adopted and determined abundances for all assumed rotation velocities.

To estimate the extent to which the uncertainties in the atmospheric parameters influence the determined abundances, we considered a synthetic spectrum calculated for $T_{\text {eff }}=12000 \mathrm{~K}$, $\log g=4.0, \xi=2 \mathrm{~km} \mathrm{~s}^{-1}$, and $V \sin i=50 \mathrm{~km} \mathrm{~s}^{-1}$. The abundances were derived based on this spectrum and by assuming atmospheric models that deviate by $\Delta T_{\text {eff }}= \pm 1000 \mathrm{~K}, \Delta \log g=$ $\pm 0.2 \mathrm{dex}$, and $\Delta \xi= \pm 1 \mathrm{~km} \mathrm{~s}^{-1}$ from the reference values (the other two parameters were kept frozen when one was allowed to vary). Comparison of the results obtained with the reference atmosphere model allows us to assess the effect of typical errors in the effective temperature, surface gravity and microturbulence on the determined abundances.

We present in Fig. A.2 the differences between the input and calculated abundances, assuming that the adopted atmospheric parameters $\left(T_{\text {eff }}, \log g, \xi\right)$ chosen are incorrect. An error in the effective temperature of $1000 \mathrm{~K}$ has the largest effect on the determined chemical abundances. In this case, the discrepancies can exceed 0.5 dex because the abundances are based on trace ions that are very sensitive to changes in $T_{\text {eff }}$. The proper determination of $T_{\text {eff }}$ is crucial to obtain the correct abundance pattern of the star. For $\Delta \log g= \pm 0.2$ and $\Delta \xi= \pm 1 \mathrm{~km} \mathrm{~s}^{-1}$, the effect is much smaller and the differences are typically below 0.1 dex.

\section{References}

Abt, H. A. 1985, ApJS, 59, 95

Abt, H. A. 2005, AJ, 629, 507

Abt, H. A., \& Morrell, N. I. 1995, ApJS, 99, 135

Abt, H. A., \& Boonyarak, C. 2004, AJ, 616, 562

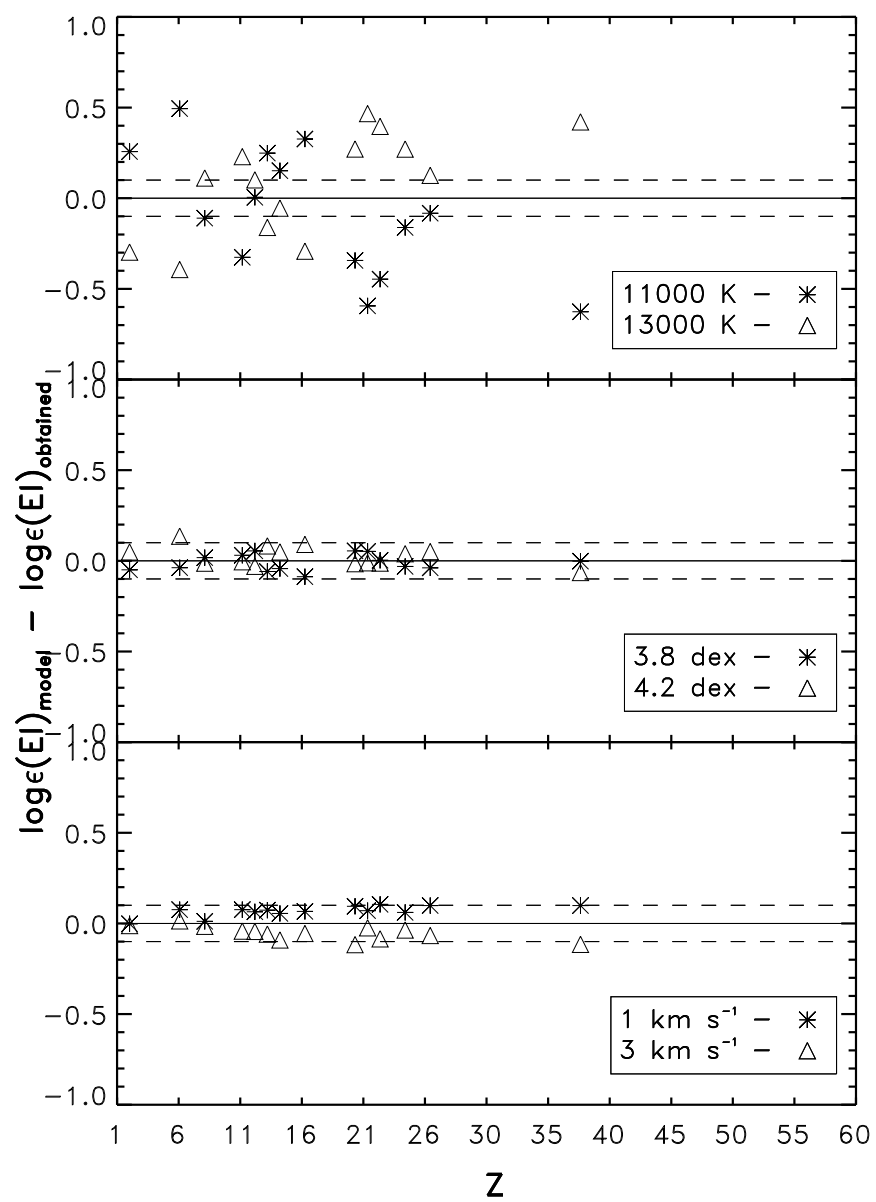

Fig. A.2. Differences between adopted and determined abundances assuming atmospheric models with an incorrect effective temperature (top panel), surface gravity (middle panel) or microturbulence (bottom panel).

Abt, H. A., Levato, H., \& Grosso, M. 2002, ApJ, 573, 359

Adelman, S. J. 1984, A\&AS, 58, 585

Adelman, S. J. 1991, MNRAS, 252, 116

Adelman, S. J., \& Fuhr, J. R. 1985, A\&A, 152, 434

Adelman, S. J., Cowley, C. R., Leckrone, D. S., Roby, S. W., \& Wahlgren, G. M. 1993, ApJ, 419, 276

Adelman, S. J., Proffitt, C. R., Wahlgren, G. M., et al. 2004, ApJS, 155, 179

Auvergne, M., Bodin, P., Boisnard, L., et al. 2009, A\&A, 506, 411

Bagnulo, S., Landstreet, J. D., Mason, E., et al. 2006, A\&A, 450, 777

Baranne, A., Queloz, D., Mayor, M., et al. 1996, A\&AS, 119, 373

Bertaud, C. 1959, JO, 42, 45

Bevington, P. R. 1969, in Data reduction and error analysis for the physical sciences (New York: McGraw-Hill)

Bychkov, V. D., Bychkova, L. V., \& Madej, J. 2003, A\&A, 407, 631

Castelli, F., \& Hubrig, S. 2004, A\&A, 425, 263

Catalano, F. A., \& Renson, P. 1998, A\&AS, 127, 421

Catanzaro, G., Leone, F., \& Catalano, F. 1999, A\&AS, 134, 211

Cenarro, A. J., Peletier, R. F., Sánchez-Blázquez, P., et al. 2007, MNRAS, 374, 664

Cowley, A. P., \& Crawford, D. L. 1971, PASP, 83, 296

Diaz-Cordoves, J., Claret, A., \& Gimenez, A. 1995, A\&AS, 110, 329

Dommanget, J., \& Nys, O. 2000, A\&A, 363, 991

Dommanget, J., \& Nys, O. 2002, Observations et Travaux, 54, 5

Durrant, C. J. 1970, MNRAS, 147, 59

Fabricius, C., Høg, E., Makarov, V. V., et al. 2002, A\&A, 384, 180

Folsom, C. P., Wade, G. A., Bagnulo, S., \& Landstreet, J. D. 2007, MNRAS, 376,361

Frankowski, A., Jancart, S., \& Jorissen, A. 2007, A\&A, 464, 377

Frémat, Y., Neiner, C., Hubert, A. M., et al. 2006, A\&A, 451, 1053

Gies, D. R., \& Lambert, D. L. 1992, ApJ, 387, 673

Gigas, D. 1988, A\&A, 192, 264

Glagolevskij, Yu. V. 1994, BSAO, 38, 152 
Gray, D. F. 2005, The Observation an Analysis of Stellar Photospheres (Cambridge University Press), The Edinburgh Building, Cambridge, CB2 2Ru, UK

Grevesse, N., Asplund, M., \& Sauval, A. J. 2007, SSRv, 130, 105

Hartkopf, W. I., Mason, B. D., McAlister, H. A., et al. 2000, AJ, 119, 3084

Hauck, B., \& Mermilliod, M. 1998, A\&AS, 129, 431

Hempel, M., \& Holweger, H. 2003, A\&A, 408, 1065

Horch, E. P., Davidson, J. W., Van Altena, W. F., et al. 2006, AJ, 131, 1000

Huang, W., \& Gies, D. R. 2006, ApJ, 648, 580

Jeffery, V. M., Woolf, D. L., \& Pollacco, D. L. 2001, A\&A, 376, 497

Jenkner, H., \& Maitzen, H. M. 1987, A\&AS, 71, 255

Jomaron, C. M., Dworetsky, M. M., \& Allen, C. S. 1999, MNRAS, 303, 555

Kaufer, A., Stahl, O., Tubbesing, S., et al. 2000, SPIE, 4008, 459

Kazarovets, E. V., Samus, N. N., Durlevich, O. V., et al. 1999, IBVS, 4659, 1

Kharchenko, N. V. 2001, KFNT, 17, 409

Koen, C., \& Eyer, L. 2002, MNRAS, 331, 45

Kudryavtsev, D. O., Romanyuk, I. I., Elkin, V. G., \& Paunzen, E. 2006, MNRAS, 372, 1804

Kukarkin, B. V., Kholopov, P. N., Kukarkina, N. P., et al. 1972, IBVS, 717, 1

Künzli, M., North, P., Kurucz, R. L., \& Nicolet, B. 1997, A\&AS, 122, 51

Kupka, F. G., Ryabchikova, T. A., Piskunov, N. E., et al. 2000, BaltA, 9, 590

Kurucz, R. 1993, CD-ROM 18

Lefever, K. 2007, Ph.D. Thesis, K.U. Leuven, Belgium

Lefever, K., Puls, J., \& Aerts, C. 2006, A\&A, 455, 259

Leone, F., \& Manfre, M. 1996, A\&A, 315, 526

Leone, F., Lanzafame, A. C., \& Pasquini, L. 1995, A\&A, 293, 457

Levato, H., \& Malaroda, S. 1974, AJ, 79, 890

Makarov, V. V., \& Kaplan, G. H. 2005, AJ, 129, 2420

Mason, B. D., Wycoff, G. L., Hartkopf, W. I., Douglass, G. G., \& Worley, C. E. 2001, AJ, 122, 466

Moon, T., \& Dworetsky, M. M. 1985, MNRAS, 217, 305

Morel, T., \& Aerts, C. 2007, CoAst, 150, 201

Morel, T., Butler, K., Aerts, C., et al. 2006, A\&A, 457, 651

Morel, T., Hubrig, S., \& Briquet, M. 2008, A\&A, 481, 453

Napiwotzki, R., Schoenberner, D., \& Wenske, V. 1993, A\&A, 268, 653
Neiner, C., Hubert, A. M., \& Catala, C. 2005, ApJS, 156, 237

Netopil, M., Paunzen, E., Maitzen, H. M., et al. 2008, A\&A, 491, 545

Niemczura, E., \& Połubek, G. 2006, ESASP, 624, 120

North, P. 1987, A\&AS, 69, 371

North, P. 1992, Stellar Magnetism, Proceedings of international meeting on the problem, Physics and evolution of stars, ed. Yu. V. Glagolevskij, \& I. I. Romanyuk (Sankt Petersburg: NAUKA), Sankt-Petersburg branch, 72

North, P., \& Kroll, R. 1989, A\&AS, 78, 325

Paunzen, E., Kamp, I., Iliev, I. Kh. et al. 1999, A\&A, 345, 597

Paunzen, E., Duffee, B., Heiter, U., et al. 2001, A\&A, 373, 625

Perryman, M. A. C., \& ESA 1997, The HIPPARCOS and TYCHO catalogues, ESA-SP 1200, ESA Publications Division

Przybilla, N., \& Butler, K. 2001, A\&A, 379, 955

Przybilla, N., Butler, K., Becker, S. R., Kudritzki, R. P., \& Venn, K. A. 2000, A\&A, 359, 1085

Roby, S. W., \& Lambert, D. L. 1990, ApJS, 73, 67

Ryabchikova, T. A. 2005, AstL, 31, 388

Sargent, A. I., Greenstein, J. L., \& Sargent, W. L. 1969, ApJ, 157, 757

Sbordone, L. 2005, MSAIS, 8, 61

Searle, L., Lungershausen, W. T., \& Sargent, W. L. 1966, ApJ, 145, 652

Semenko, E. A., Kudryavtsev, D. O., Ryabchikova, T. A., et al. 2008, AstBu, 63, 128

Smith, K. C. 1993, A\&A, 276, 393

Smith, K. C. 1994, A\&A, 291, 521

Smith, K. C. 1996, Ap\&SS, 237, 77

Smith, K. C. 1997, A\&A, 319, 928

Smith, K. C., \& Dworetsky, M. M. 1993, A\&A, 274, 335

Solano, E., Catala, C., Garrido, R., et al. 2005, AJ, 129, 547

Sowell, J. R., \& Wilson, J. W. 1993, PASP, 105, 36

Takeda, Y. 1995, PASJ, 47, 287

Vogt, N., \& Faundez, A. M. 1979, A\&AS, 36, 477

Wade, R. A., \& Rucinski, S. M. 1995, A\&AS, 60, 471

Walther, M. E. 1949, ApJ, 110, 67

Whitford, A. E. 1963, Reports of Observatories 1962-63, Lick Observatory, Stellar Spectra, 68, 640 
Table 1. The description of the analysed stars and their observations. The observation time relates to the beginning of the exposure.

\begin{tabular}{|c|c|c|c|c|c|c|c|c|}
\hline HD & Sp. type & "Spectrograph & $\begin{array}{c}\text { Obs. date } \\
{[\mathrm{UT}]}\end{array}$ & $\begin{array}{c}\text { Obs. time } \\
{[\mathrm{UT}]}\end{array}$ & $\begin{array}{c} \\
{[\mathrm{mag}]}\end{array}$ & $\begin{array}{c}E(B-V) \\
{[\mathrm{mag}]}\end{array}$ & "Binarity & ב Abundance analysis \\
\hline 42677 & $\mathrm{~B} 8$ & FEROS & $2002-01-27$ & $03: 37: 43$ & 7.79 & 0.06 & & \\
\hline 43406 & B9 & ELODIE & 2003-01-27 & $18: 40: 31$ & 7.16 & 0.00 & & \\
\hline 43461 & B6V & FEROS & $2002-01-28$ & $00: 40: 32$ & 6.63 & 0.09 & & \\
\hline 43743 & B9 & FEROS & $2002-01-28$ & $00: 57: 28$ & 7.70 & 0.00 & & \\
\hline 44321 & B9 & FEROS & 2003-01-15 & 02:41:10 & 7.64 & 0.01 & & \\
\hline 44354 & B9 & FEROS & 2003-01-17 & $01: 40: 47$ & 7.70 & 0.01 & & \\
\hline 44720 & B8 & FEROS & 2003-01-17 & 01:58:58 & 7.22 & 0.01 & & \\
\hline 44948 & $\mathrm{~B} 8 \mathrm{Vp}$ & FEROS & 2003-01-17 & 02:05:02 & 6.73 & 0.02 & $3,4,5,6,7,8$ & \\
\hline 45050 & B9V & FEROS & $2003-01-15$ & 03:11:35 & 6.66 & 0.02 & $3,4,5,6,9,10,11$ & \\
\hline 45153 & B8 & FEROS & 2003-01-15 & $03: 16: 54$ & 7.30 & 0.01 & & \\
\hline 45397 & B8 & FEROS & 2003-01-14 & $03: 40: 40$ & 7.81 & 0.01 & $3,5,6$ & \\
\hline 45515 & $\mathrm{~B} 8 \mathrm{~V}$ & FEROS & 2003-01-17 & 02:48:56 & 7.88 & 0.01 & & \\
\hline 45516 & B9 & FEROS & 2003-01-17 & $02: 56: 49$ & 7.82 & 0.01 & & \\
\hline 45563 & B9V & FEROS & 2003-01-15 & $03: 57: 36$ & 6.48 & 0.00 & 5,6 & \\
\hline 45583 & B8 & FEROS & 2003-01-17 & 03:11:31 & 7.98 & 0.01 & & 14,15 \\
\hline 45657 & B9 & FEROS & $2002-01-28$ & $02: 26: 30$ & 7.89 & 0.00 & & \\
\hline 45709 & B9 & FEROS & 2003-01-16 & $01: 05: 41$ & 7.55 & 0.00 & & \\
\hline 45760 & B9.5V & FEROS & 2003-01-15 & 04:08:40 & 7.56 & 0.00 & & \\
\hline 45975 & B9 & FEROS & 2003-01-16 & 01:29:11 & 7.46 & 0.03 & & \\
\hline 46138 & B9 & FEROS & 2003-01-14 & $04: 20: 52$ & 7.46 & 0.00 & & \\
\hline 46179 & B9V & ELODIE & 2003-01-16 & $19: 26: 53$ & 6.69 & 0.02 & $3,4,5,6$ & \\
\hline 46340 & B8 & FEROS & $2002-01-28$ & 03:27:09 & 7.65 & 0.00 & $3,4,6$ & \\
\hline 46541 & B9 & FEROS & $\begin{array}{l}2003-01-16 \\
2003-01-18\end{array}$ & $\begin{array}{l}01: 41: 58 \\
00: 54: 29\end{array}$ & 7.90 & 0.03 & & \\
\hline 46885 & B9III & ELODIE & $2003-01-16$ & $20: 27: 27$ & 6.55 & 0.02 & & \\
\hline 46886 & B9 & FEROS & $2002-01-28$ & 03:43:57 & 7.95 & 0.01 & & \\
\hline 47022 & B9 & ELODIE & $\begin{array}{l}2003-01-16 \\
2003-01-15\end{array}$ & $\begin{array}{l}22: 19: 10 \\
21: 11: 44\end{array}$ & 7.74 & 0.00 & & \\
\hline 47221 & B9 & ELODIE & $2003-01-27$ & 00:00:45 & 7.81 & 0.00 & & \\
\hline 47257 & B9 & ELODIE & 2003-01-18 & $19: 45: 23$ & 7.26 & 0.00 & & \\
\hline 47272 & B9V & ELODIE & $2003-01-24$ & 20:06:06 & 7.62 & 0.03 & & \\
\hline 47278 & B9 & FEROS & $2002-01-28$ & $04: 45: 28$ & 7.23 & 0.07 & & \\
\hline 47431 & B8IIIn & ELODIE & 2003-01-16 & $20: 44: 13$ & 6.57 & 0.03 & & \\
\hline 47756 & B8IIIs & ELODIE & 2003-01-15 & $23: 42: 53$ & 6.51 & 0.00 & & \\
\hline 47759 & B9 & ELODIE & $2003-01-22$ & $21: 27: 32$ & 7.52 & 0.00 & & \\
\hline 47964 & B8III & FEROS & 2003-01-15 & $05: 17: 48$ & 5.79 & 0.00 & $3,4,5,6$ & \\
\hline 48212 & B9 & ELODIE & $2003-01-26$ & $23: 17: 17$ & 7.80 & 0.04 & $4,5,6$ & \\
\hline 48497 & B8 & FEROS & 2003-01-16 & $03: 22: 30$ & 7.50 & 0.00 & & \\
\hline 48808 & B9 & FEROS & 2003-01-14 & $05: 18: 29$ & 7.45 & 0.04 & & \\
\hline 48957 & B9 & FEROS & 2003-01-15 & $05: 54: 50$ & 8.00 & 0.03 & $4,5,6$ & \\
\hline 49123 & B9 & FEROS & 2003-01-17 & 03:32:18 & 7.22 & 0.02 & & \\
\hline 49481 & B8 & FEROS & 2003-01-18 & 04:07:10 & 6.80 & 0.04 & & \\
\hline 49643 & B8IIIn & FEROS & 2003-01-16 & $05: 06: 34$ & 5.75 & 0.00 & $3,4,5,6,9,12$ & \\
\hline 49711 & B8V & ELODIE & 2003-01-18 & $20: 52: 53$ & 7.43 & 0.06 & & \\
\hline 49713 & B9p & FEROS & 2003-01-15 & $06: 24: 29$ & 7.32 & 0.00 & & \\
\hline 49886 & B8 & FEROS & 2003-01-17 & $03: 52: 32$ & 7.58 & 0.00 & & \\
\hline 49935 & B8 & FEROS & 2003-01-16 & 05:11:05 & 6.86 & - & & \\
\hline 50251 & B8V & FEROS & 2003-01-18 & $04: 25: 34$ & 7.20 & 0.01 & & \\
\hline 50252 & B9V & FEROS & 2003-01-17 & 04:06:13 & 7.95 & 0.07 & & \\
\hline 50513 & B8 & FEROS & 2003-01-17 & $04: 21: 58$ & 7.82 & 0.00 & & \\
\hline 50751 & B8 & FEROS & 2003-01-17 & $04: 37: 56$ & 7.90 & 0.00 & & \\
\hline 51079 & B8V & FEROS & 2003-01-18 & $05: 12: 03$ & 7.94 & 0.04 & & \\
\hline 52312 & B9III & FEROS & 2003-01-18 & $05: 35: 03$ & 5.96 & 0.01 & $3,4,5,6$ & \\
\hline 53004 & B9 & FEROS & 2003-01-14 & 07:00:14 & 7.26 & 0.03 & $3,4,5,6$ & \\
\hline 53083 & B8 & FEROS & 2003-01-15 & $07: 24: 17$ & 7.10 & 0.02 & & \\
\hline 53851 & B9 & FEROS & 2003-01-14 & 07:21:06 & 7.58 & 0.00 & & \\
\hline 54929 & B9 & FEROS & 2003-01-18 & $06: 42: 53$ & 7.34 & 0.02 & $3,4,5,6$ & \\
\hline 55362 & B9 & FEROS & 2003-01-14 & 07:58:01 & 7.95 & 0.00 & & \\
\hline 55793 & B8 & FEROS & 2003-01-14 & 08:07:45 & 7.86 & 0.01 & & \\
\hline 56006 & B8 & FEROS & 2003-01-17 & 07:04:32 & 7.69 & 0.06 & & \\
\hline 56446 & B8III & FEROS & 2003-01-15 & 08:11:38 & 6.65 & 0.00 & & \\
\hline 56610 & B9 & FEROS & 2003-01-17 & 07:19:19 & 7.80 & 0.00 & & \\
\hline 56613 & B9 & FEROS & 2003-01-18 & $07: 24: 51$ & 7.10 & 0.00 & & \\
\hline
\end{tabular}


Table 1. continued.

\begin{tabular}{|c|c|c|c|c|c|c|c|c|}
\hline HD & Sp. type & Spectrograph & $\begin{array}{c}\text { Obs. date } \\
\text { [UT] }\end{array}$ & $\begin{array}{l}\text { Obs. time } \\
\text { [UT] }\end{array}$ & $\begin{array}{c}V \\
{[\mathrm{mag}]}\end{array}$ & $\begin{array}{c}E(B-V) \\
{[\mathrm{mag}]}\end{array}$ & Binarity & Abundance analysis \\
\hline 57275 & B9 & ELODIE & 2001-11-28 & 03:24:06 & 6.90 & 0.00 & $3,4,6,5,7$ & \\
\hline 57293 & B9III-IV & FEROS & 2003-01-16 & $07: 54: 23$ & 7.99 & 0.03 & $3,4,6,5,7,12$ & \\
\hline 168202 & B9 & ELODIE & 2002-08-15 & 19:37:44 & 7.50 & 0.00 & & \\
\hline 168932 & B9 & FEROS & 2001-07-05 & $02: 56: 50$ & 7.28 & 0.00 & 1,2 & \\
\hline 169224 & B9 & ELODIE & 2002-08-16 & $20: 11: 21$ & 7.50 & 0.04 & & \\
\hline 169225 & B9 & FEROS & 2001-07-05 & 03:06:33 & 7.72 & 0.01 & & \\
\hline 169512 & B9 & ELODIE & 2002-08-19 & 19:36:33 & 7.91 & 0.15 & $3,4,5,6,7$ & \\
\hline 169578 & B9V & FEROS & 2001-07-06 & $07: 28: 52$ & 6.73 & 0.09 & 1,2 & \\
\hline 170783 & B5 & ELODIE & $\begin{array}{l}2000-06-18 \\
2000-06-18\end{array}$ & $\begin{array}{l}00: 38: 18 \\
00: 57: 09\end{array}$ & 7.73 & 0.34 & & \\
\hline 170935 & B8 & ELODIE & 2002-08-18 & 19:27:06 & 7.38 & 0.20 & & \\
\hline 171247 & B8IIIsp & FEROS & 2001-07-07 & $04: 42: 34$ & 6.42 & 0.07 & $3,4,5,6$ & 13 \\
\hline 171931 & B9 & FEROS & 2005-06-18 & 02:07:17 & 9.19 & 0.26 & & \\
\hline 172850 & B9 & FEROS & 2001-07-05 & 04:10:15 & 7.75 & 0.08 & & \\
\hline 173673 & B8 & FEROS & 2001-07-05 & 04:43:37 & 7.65 & 0.13 & & \\
\hline 174701 & B9 & FEROS & 2001-07-05 & 06:07:22 & 8.00 & 0.15 & $4,5,6$ & \\
\hline 174836 & B9 & FEROS & 2001-07-05 & $06: 22: 37$ & 7.89 & 0.00 & & \\
\hline 174884 & B8 & ELODIE & $\begin{array}{l}2000-06-10 \\
2000-06-10\end{array}$ & $\begin{array}{l}01: 24: 45 \\
02: 06: 56\end{array}$ & 7.99 & 0.00 & & \\
\hline 176076 & B9 & FEROS & 2001-07-05 & $07: 19: 25$ & 7.28 & 0.06 & & \\
\hline 176158 & B9 & FEROS & 2001-07-05 & 07:39:42 & 7.52 & 0.22 & & \\
\hline 176258 & B9V & FEROS & 2001-07-07 & 05:44:09 & 7.52 & 0.00 & & \\
\hline 177756 & B9Vn & FEROS & 2001-07-06 & $02: 32: 47$ & 3.43 & 0.00 & & \\
\hline 177880 & B5V & FEROS & 2001-07-06 & $04: 23: 07$ & 6.76 & 0.26 & $3,4,5,6$ & \\
\hline 178744 & $\mathrm{~B} 5 \mathrm{Vn}$ & FEROS & 2001-07-05 & $09: 28: 56$ & 6.33 & 0.00 & & \\
\hline 179124 & B9V & FEROS & 2001-07-06 & $09: 12: 42$ & 6.90 & 0.04 & & \\
\hline 179761 & B8II-III & FEROS & 2001-07-07 & 06:03:24 & 5.14 & 0.05 & $3,4,5,6$ & $\begin{array}{l}16,17,18,19,20,21,22, \\
23,24,25,26,27,28,29, \\
30,31\end{array}$ \\
\hline 181440 & B9III & FEROS & 2001-07-05 & 09:44:56 & 5.48 & 0.02 & & \\
\hline 181761 & B8 & FEROS & 2001-07-04 & 09:34:12 & 7.94 & 0.01 & & \\
\hline 182198 & B9V & FEROS & 2001-07-06 & 04:35:39 & 7.94 & 0.04 & & \\
\hline
\end{tabular}

References: (1) Frankowski et al. (2007); (2) Makarov et al. (2005); (3) Dommanget \& Nys (2000); (4) Dommanget \& Nys (2002); (5) Fabricius et al. (2002); (6) Mason et al. (2001); (7) Abt (1985); (8) Horch et al. (2006); (9) Sowell \& Wilson (1993); (10) Abt \& Boonyarak (2004); (11) Abt (2005); (12) Hartkopf et al. (2000); (13) Leone \& Manfre (1996); (14) Semenko et al. (2008); (15) Levato \& Malaroda (1974); (16) Searle et al. (1966); (17) Sargent et al. (1969); (18) Durrant (1970); (19) Adelman (1984); (20) Adelman \& Fuhr (1985); (21) Adelman (1991); (22) Roby \& Lambert (1990); (23) Adelman et al. (1993); (24) Smith (1993); (25) Smith \& Dworetsky (1993); (26) Smith (1994); (27) Smith (1996); (28) Smith (1997); (29) Adelman et al. (2004); (30) Ryabchikova (2005); (31) Cenarro et al. (2007). 
Table 2. The atmospheric parameters, abundances of chemical elements and rotation velocities of all analysed stars.

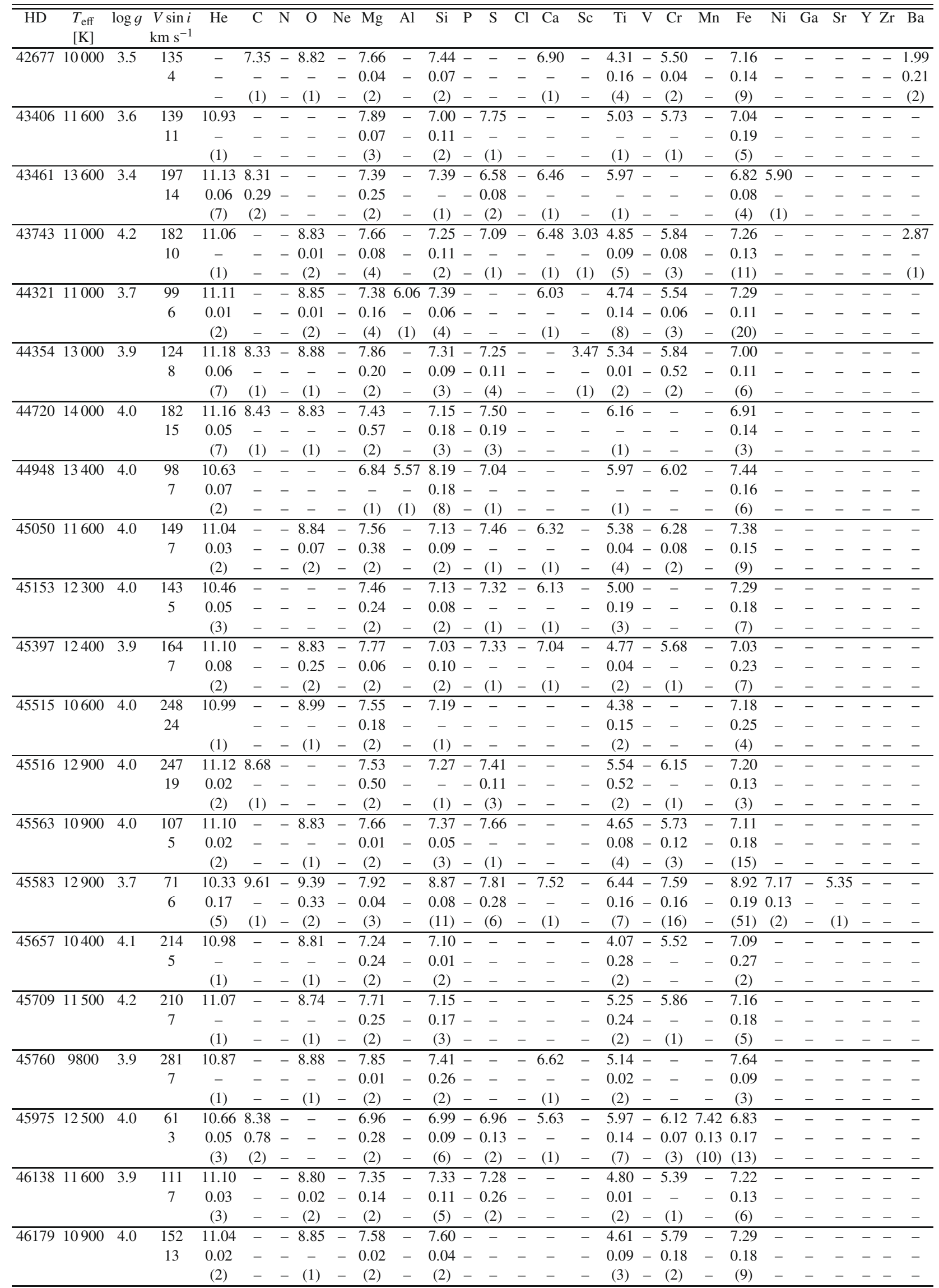


Table 2. continued.

\begin{tabular}{|c|c|c|c|c|c|c|c|c|c|c|c|c|c|c|c|c|c|c|c|c|c|c|c|c|c|c|}
\hline HD & $\begin{array}{l}T_{\text {eff }} \\
{[\mathrm{K}]}\end{array}$ & $\bar{g} g$ & $\begin{array}{c}V \sin i \\
\mathrm{~km} \mathrm{~s}^{-1}\end{array}$ & $\mathrm{He}$ & $\bar{C}$ & $\mathrm{~N}$ & $\mathrm{O}$ & $\mathrm{Ne}$ & $\overline{M g}$ & $\mathrm{Al}$ & $\mathrm{Si}$ & $\bar{P}$ & $\bar{S}$ & $\mathrm{Cl}$ & $\mathrm{Ca}$ & $\mathrm{Sc}$ & $\mathrm{Ti}$ & $\overline{\mathrm{V}}$ & $\mathrm{Cr}$ & $\mathrm{Mn}$ & $\mathrm{Fe}$ & $\mathrm{Ni}$ & $\mathrm{Ga}$ & $\mathrm{Sr}$ & $\bar{Y}$ & $\begin{array}{ll}\mathrm{Zr} & \mathrm{Ba}\end{array}$ \\
\hline \multirow[t]{3}{*}{46340} & 9500 & 3.9 & 128 & 10.61 & - & - & 8.83 & - & 8.02 & - & 7.61 & - & - & - & 6.77 & 3.43 & 5.26 & 4.27 & 5.97 & 5.55 & 7.64 & - & - & 2.77 & - & -2.67 \\
\hline & & & 4 & 0.02 & - & - & 0.01 & - & 0.16 & - & 0.10 & - & - & - & 0.19 & 0.11 & 0.15 & - & 0.07 & - & 0.09 & - & - & - & - & - \\
\hline & & & & (2) & - & - & (2) & - & (5) & - & (2) & - & - & - & (2) & (2) & (13) & (1) & (4) & (1) & (16) & - & - & (1) & - & $-(1)$ \\
\hline \multirow[t]{3}{*}{46541} & 9600 & 3.5 & 58 & - & 7.99 & - & 8.73 & - & 7.52 & 6.45 & 7.38 & - & - & - & 5.47 & 2.90 & 4.79 & - & 5.70 & 4.81 & 7.29 & - & - & - & - & -2.56 \\
\hline & & & & - & 0.57 & - & 0.01 & - & 0.05 & - & 0.11 & 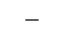 & - & - & 0.75 & 0.08 & 0.06 & - & 0.08 & - & 0.08 & - & - & - & 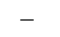 & -0.11 \\
\hline & & & & & (2) & - & (2) & $\begin{array}{lll}- & \end{array}$ & (4) & (1) & (5) & - & - & - & (2) & (4) & (17) & - & (12) & (1) & (34) & - & - & - & - & $-\quad(2)$ \\
\hline \multirow[t]{3}{*}{46885} & 11700 & 3.6 & 32 & 10.86 & - & - & 8.70 & - & 6.49 & - & 6.52 & - & - & - & - & - & 4.15 & - & 4.91 & - & 6.65 & - & - & - & - & - \\
\hline & & & 1 & 0.04 & - & - & - & - & 0.07 & - & 0.12 & . & - & - & - & - & 0.08 & - & 0.13 & - & 0.06 & - & - & - & - & - \\
\hline & & & & (3) & - & - & (1) & - & (2) & - & (5) & - & - & - & - & - & (3) & - & (2) & - & (16) & - & - & - & - & - \\
\hline \multirow[t]{3}{*}{46886} & 12900 & 3.8 & 18 & 10.56 & 7.84 & 7.42 & 8.54 & 7.87 & 6.52 & 6.33 & 6.63 & 5.73 & 6.67 & 5.14 & 6.47 & 4.00 & 5.53 & - & 6.30 & 7.37 & 6.66 & 6.09 & 5.85 & - & - & - \\
\hline & & & 1 & 0.06 & 0.16 & 0.55 & - & - & 0.10 & - & 0.17 & 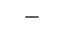 & 0.40 & - & - & 0.01 & 0.14 & - & 0.11 & 0.13 & 0.11 & 0.13 & - & - & - & - \\
\hline & & & & (4) & (2) & (2) & (1) & (1) & (2) & (1) & (8) & (1) & (8) & (1) & (1) & (2) & (16) & - & (19) & (23) & (23) & (2) & (1) & - & - & - \\
\hline 47022 & 13000 & 3.8 & 123 & 10.98 & - & - & 8.69 & - & 7.58 & - & 7.26 & - & 7.46 & - & - & - & 5.20 & - & 5.65 & - & 7.19 & - & - & - & - & - \\
\hline & & & 8 & 0.07 & - & - & - & - & - & 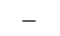 & 0.08 & 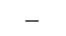 & 0.17 & - & - & - & - & - & 0.03 & - & 0.18 & - & - & - & - & - \\
\hline & & & & (3) & - & - & (1) & - & (1) & - & (2) & - & (2) & - & - & - & (1) & - & (2) & - & (4) & - & - & - & - & - \\
\hline 77221 & 13800 & 4.0 & 189 & $\begin{array}{ll}11.10 \\
\end{array}$ & - & - & - & - & 8.03 & - & 7.33 & - & 6.75 & - & - & - & 5.51 & - & - & - & 7.18 & - & - & - & - & - \\
\hline & & & 13 & 0.01 & - & - & - & - & 0.07 & - & 0.15 & - & 62 & - & - & - & 0.14 & - & - & - & 0.36 & - & - & - & - & - \\
\hline & & & & (2) & - & $\begin{array}{ll}- \\
-\end{array}$ & - & - & (2) & - & (2) & $\begin{array}{lll}- & \\
-1\end{array}$ & (2) & - & - & - & (2) & - & - & - & (3) & - & - & - & - & - \\
\hline 47257 & 10100 & 4.0 & 48 & 10.80 & - & - & 8.57 & - & 7.52 & - & 7.18 & - & - & - & 5.83 & 2.99 & 4.53 & - & 5.42 & 4.52 & 7.07 & - & - & - & - & -2.13 \\
\hline & & & 2 & - & - & - & - & - & 0.11 & - & 0.08 & - & - & - & 0.27 & - & 0.10 & - & 0.07 & - & 0.09 & - & - & - & - & - \\
\hline & & & & (1) & - & - & (1) & - & (3) & - & (4) & - & - & - & (2) & (1) & (15) & - & (6) & (1) & (21) & - & - & - & - & $-(1)$ \\
\hline 47272 & 10000 & 4.0 & 21 & 10.74 & 8.72 & - & 8.55 & - & 7.38 & 6.66 & 7.35 & - & - & - & 6.17 & 2.77 & 4.69 & - & 5.75 & - & 7.36 & 6.42 & - & 2.84 & 67 & -2.98 \\
\hline & & & 1 & - & - & - & 0.15 & - & 0.12 & - & 0.01 & - & - & - & 0.03 & 0.19 & 0.09 & - & 0.07 & - & 0.10 & 0.28 & - & 0.09 & 0.38 & -0.04 \\
\hline & & & & (1) & (1) & - & (2) & - & (6) & (1) & (4) & - & - & - & (4) & (4) & (19) & - & (8) & - & (51) & (2) & - & (2) & (2) & $-(2)$ \\
\hline 47278 & 11500 & 4.1 & 38 & 10.73 & - & - & 8.50 & - & 7.18 & - & 7.12 & - & 7.00 & - & 6.26 & - & 5.51 & - & 6.22 & 6.55 & 7.07 & - & - & - & - & - \\
\hline & & & 2 & 0.06 & - & - & - & - & 0.03 & - & 0.10 & - & - & - & - & - & 0.13 & - & 0.09 & - & 0.19 & - & - & - & - & - \\
\hline & & & & (4) & - & $\begin{array}{lll}- & \\
-1\end{array}$ & (1) & - & (2) & - & (4) 7 - & (1) & - & (1) & - & (15) & - & (6) & (1) & (19) & - & - & - & - & $\begin{array}{ll}- \\
-\end{array}$ & - \\
\hline 47431 & 12200 & 3.6 & 110 & 11.16 & - & - & 8.87 & - & 7.99 & - & 7.35 & - & - & - & - & - & 5.03 & - & 5.53 & - & 7.28 & - & - & - & - & - \\
\hline & & & 9 & 0.01 & - & - & 0.19 & - & 0.17 & - & 0.19 & - & - & - & - & - & 0.12 & - & - & - & 0.08 & - & . & 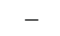 & - & - \\
\hline & & & & (2) & - & - & (2) & - & (2) & - & 3) & - & - & - & - & - & (2) & - & (1) & - & (8) & - & - & - & - & - \\
\hline 47756 & 13300 & 3.6 & 27 & 10.25 & 8.67 & - & 8.90 & - & 7.49 & 5.40 & 8.21 & 5.42 & 6.90 & - & - & - & 6.73 & - & 7.00 & - & 8.45 & 5.95 & - & 4.30 & - & - \\
\hline & & & 1 & 0.12 & - & - & - & - & 0.20 & - & 0.04 & 0.13 & 0.08 & - & - & - & 0.20 & - & 0.12 & - & 0.10 & - & - & - & - & - \\
\hline & & & & (2) & (1) & - & (1) & - & (2) & (1) & (6) & (2) & (4) & - & - & - & (8) & - & (12) & - & (46) & (1) & - & (1) & - & - \\
\hline 47759 & 10200 & 3.9 & 100 & 11.18 & - & - & 8.23 & - & 7.58 & - & - & - & - & - & - & - & 5.66 & - & 7.59 & - & 8.39 & - & - & - & - & - \\
\hline & & & 4 & - & - & - & - & - & - & 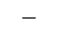 & 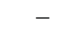 & - & - & - & - & - & 0.06 & - & 0.17 & - & 0.08 & - & - & - & - & - \\
\hline & & & & (1) & - & - & (1) & - & (1) & - & - & - & - & - & - & - & (2) & - & (2) & - & (7) & - & - & - & - & - \\
\hline 47964 & 12300 & 3.2 & 54 & 10.96 & 8.16 & - & 8.83 & 7.99 & 7.30 & - & $\overline{01}$ & - & 6 & - & 6.59 & - & 5.33 & - & 5.84 & 5.71 & 7.09 & 6.22 & - & - & - & - \\
\hline & & & 3 & 0.03 & - & - & - & - & 0.04 & - & 0.04 & - & 0 & - & - & - & 0.20 & - & 0.13 & 0.25 & 0.11 & 0.2 & 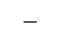 & - & - & - \\
\hline & & & & (7) & (1) & - & (1) & (1) & (3) & - & 5) & - & $(\varepsilon$ & - & (1) & - & (4) & - & (4) & (2) & (18) & (2) & - & - & - & - \\
\hline 48212 & 12000 & 3.3 & 173 & 10.98 & - & - & 8.88 & - & 7.66 & - & 7.30 & - & 6.82 & - & - & - & 5.26 & - & 5.74 & - & 7.24 & - & - & - & - & - \\
\hline & & & 7 & 0.07 & - & - & 0.05 & - & - & - & 0.06 & 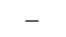 & - & - & - & - & 0.26 & - & - & - & 0.20 & - & . & - & - & - \\
\hline & & & & (2) & - & - & (2) & - & (1) & - & (2) & - & (1) & - & - & - & (2) & - & (1) & - & (6) & - & - & - & - & - \\
\hline 48497 & 14000 & 4.0 & 13 & 11.01 & 8.32 & 7.41 & 8.87 & 8.32 & 7.30 & 6.10 & 7.24 & - & 6.97 & - & 5.98 & - & 4.88 & - & 5.06 & - & 7.06 & 5.85 & - & 2.16 & - & - \\
\hline & & & 1 & 0.05 & 0.09 & 0.49 & 0.11 & 0.05 & 0.20 & - & 0.08 & - & 0.10 & - & 0.03 & - & 0.03 & - & 0.04 & - & 0.14 & 0.07 & - & - & - & - \\
\hline & & & & (5) & (3) & (2) & (3) & (2) & (3) & (1) & (8) & - & (11) & - & (2) & - & (2) & - & (2) & - & (33) & (2) & - & (1) & - & - \\
\hline 48808 & 11900 & 3.2 & 65 & 11.11 & 7.77 & - & 8.47 & - & 7.14 & - & 6.68 & - & 6. & - & - & - & 4.70 & - & 4.95 & - & 6.75 & 5.6 & - & - & - & - \\
\hline & & & 4 & 0.05 & - & - & 0.08 & - & 0.14 & - & 0.07 & - & 0. & - & - & - & 0.11 & - & 0.15 & - & 0.19 & 0.0 & - & - & - & - \\
\hline & & & & (7) & (1) & - & (2) & - & (3) & - & (7) & - & (2) & - & - & - & (5) & - & (6) & - & (17) & (2) & - & - & - & $\begin{array}{ll}- \\
-\end{array}$ \\
\hline 48957 & 12500 & 3.2 & 34 & 10.91 & 8.01 & - & - & - & 7.17 & - & 87 & - & 6.35 & - & - & $\begin{array}{ll}- \\
-\end{array}$ & 4.85 & - & 5.07 & - & 6.92 & - & - & - & - & - \\
\hline & & & 4 & 0.09 & - & - & - & - & - & - & 0.07 & - & 0.44 & - & - & $\begin{array}{lll}- & \\
-1\end{array}$ & 0.02 & - & 0.06 & - & 0.16 & - & - & - & - & - \\
\hline & & & & (4) & (1) & - & - & - & (1) & - & (4) & - & (2) & - & - & - & (2) & - & (2) & - & (13) & - & - & - & - & - \\
\hline 49123 & 10300 & 3.6 & 47 & 10.97 & 8.48 & 7.63 & 8.67 & - & 7.37 & - & 7.18 & - & - & - & 6.14 & 3.10 & 4.45 & - & 5.21 & - & 6.84 & 5.88 & - & - & 2.87 & -2.59 \\
\hline & & & 2 & 0.04 & 0.23 & - & - & - & 0.04 & - & 0.08 & - & - & - & 0.05 & - & 0.17 & - & 0.18 & - & 0.14 & - & - & - & - & - \\
\hline & & & & (2) & (2) & (1) & (1) & - & (2) & - & (5) & - & - & - & (2) & (1) & (9) & - & (4) & - & (16) & (1) & - & - & (1) & (1) \\
\hline 49481 & 12500 & 3.3 & 11 & 10.73 & 8.26 & 7.69 & 8.82 & 7.80 & 7.16 & 5.82 & 7.28 & 01 & 6.96 & - & 5.92 & 3.32 & 4.91 & - & 5.62 & 5.64 & 7.17 & 5.6 & - & 2.45 & & - \\
\hline & & & 1 & - & 0.17 & - & 0.03 & 0.18 & 0.17 & 0.27 & 11 & 0.24 & 0.16 & - & 0.12 & - & 0.12 & - & 0.10 & 0.37 & 0.11 & 0.19 & - & - & - & - \\
\hline & & & & (1) & (4) & (1) & (3) & (2) & (3) & (2) & (12) & (3) & (18) & - & (2) & (1) & (16) & - & (12) & (2) & (81) & (5) & - & (1) & - & - \\
\hline 49643 & 14100 & 4.0 & 257 & 11.09 & 8.35 & - & - & - & 7.26 & - & 7.80 & - & 8. & - & - & $\begin{array}{lll}- & \\
-\end{array}$ & 5.17 & - & $\begin{array}{lll}- & \\
-\end{array}$ & - & 6.72 & 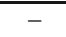 & - & - & - & - \\
\hline & & & 22 & 0.06 & - & - & - & - & 0.66 & - & - & - & 0.6 & - & - & - & 0.41 & - & - & - & 0.04 & - & - & - & - & - \\
\hline & & & & (4) & (1) & - & - & - & (2) & - & (1) & - & (2) & - & - & - & (2) & - & - & - & (2) & - & - & - & - & - \\
\hline 49711 & 11300 & 3.4 & 212 & 11.04 & - & - & 8.81 & - & 7.54 & $\begin{array}{ll}- & \\
-\end{array}$ & 7.53 & - & 7.46 & - & - & - & 4.39 & - & 5.36 & - & 7.29 & - & - & - & - & \\
\hline & & & 8 & - & - & - & - & - & 0.13 & - & 0.08 & - & - & 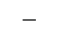 & - & - & 0.04 & - & 0.02 & - & 0.18 & - & - & - & - & - \\
\hline & & & & (1) & - & - & (1) & - & (2) & - & (2) & - & (1) & - & 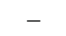 & - & (2) & - & (2) & - & (6) & - & - & - & - & - \\
\hline
\end{tabular}


Table 2. continued.

\begin{tabular}{|c|c|c|c|c|c|c|c|c|c|c|c|c|c|c|c|c|c|c|c|c|c|c|c|c|c|c|c|}
\hline$\overline{\mathrm{HD}}$ & $\begin{array}{l}T_{\text {eff }} \\
{[\mathrm{K}]}\end{array}$ & $\overline{\log g}$ & $\begin{array}{r}\sin i \\
\mathrm{~km} \mathrm{~s}^{-1}\end{array}$ & $\overline{\mathrm{He}}$ & $\overline{\overline{\mathrm{C}}}$ & $\overline{\mathrm{N}}$ & $\overline{\overline{\mathrm{O}}}$ & $\overline{\mathrm{Ne}}$ & $\overline{\mathrm{Mg}}$ & $\overline{\mathrm{Al}}$ & $\overline{\mathrm{Si}}$ & $\overline{\overline{\mathrm{P}}}$ & $\overline{\bar{S}}$ & $\overline{\overline{\mathrm{Cl}}}$ & $\overline{\mathrm{Ca}}$ & $\overline{\mathrm{Sc}}$ & $\overline{\overline{\mathrm{Ti}}}$ & $\overline{\bar{V}}$ & $\overline{\mathrm{Cr}}$ & 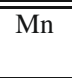 & $\overline{\overline{\mathrm{Fe}}}$ & $\overline{\mathrm{Ni}}$ & $\overline{\mathrm{Ga}}$ & $\overline{\mathrm{Sr}}$ & $\overline{\bar{Y}}$ & $\overline{\mathrm{Zr}}$ & $\overline{\overline{\mathrm{Ba}}}$ \\
\hline \multirow[t]{3}{*}{49713} & 12000 & 3.8 & 50 & 10.66 & - & - & 8.76 & - & 6.55 & - & 8.46 & - & 7.61 & - & 6.61 & 3.31 & 5.78 & - & 7.85 & 6.74 & 8.50 & - & - & - & - & - & - \\
\hline & & & 2 & 0.39 & - & - & 0.03 & - & - & - & 0.10 & - & 0.38 & - & 0.02 & 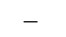 & 0.20 & - & 0.19 & 0.13 & 0.13 & - & - & - & & . & - \\
\hline & & & & (2) & - & - & (2) & - & (1) & - & (5) & - & (2) & - & (2) & (1) & (8) & - & (26) & (2) & (27) & - & - & - & - & - & - \\
\hline \multirow[t]{3}{*}{49886} & 13000 & 4.0 & 11 & - & 7.94 & - & 8.74 & - & 6.95 & 5.10 & 7.50 & 7.30 & 5.72 & - & 6.66 & - & 5.33 & - & 5.94 & - & 8.34 & 6.88 & - & - & - & - & - \\
\hline & & & 1 & - & . & - & 0.05 & - & 0.19 & - & 0.07 & 0.09 & 0.30 & - & 0.06 & 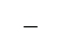 & 0.18 & - & 0.10 & - & 0.09 & 0.12 & - & - & - & - & - \\
\hline & & & & & (1) & - & (3) & - & (2) & (1) & (11) & (2) & (3) & - & (2) & - & (14) & - & (12) & - & (177) & (4) & - & - & - & - & - \\
\hline \multirow[t]{3}{*}{49935} & 15200 & 4.0 & 100 & 10.59 & 7.43 & - & - & - & 7.59 & - & 6.86 & - & 6.74 & - & 4.68 & - & - & - & - & - & 7.49 & - & - & - & - & - & - \\
\hline & & & 14 & 0.03 & - & - & - & - & 0.03 & - & 0.09 & - & 0.17 & - & 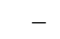 & - & - & - & - & - & 0.19 & - & - & - & - & - & - \\
\hline & & & & (5) & (1) & - & - & - & (2) & - & (3) & - & (5) & - & (1) & - & - & - & - & - & (8) & - & - & - & - & - & - \\
\hline 50251 & 12000 & 3.2 & 15 & 10.92 & 8.32 & 7.81 & 8.73 & 8.02 & 7.54 & 6.06 & 7.30 & 5.60 & 6.91 & - & 6.37 & - & 4.55 & 4.22 & 5.57 & 5.99 & 7.20 & 6.09 & - & 2.58 & 3.09 & - & $\overline{2.24}$ \\
\hline & & & 1 & 0.06 & 0.09 & 0.06 & 0.12 & 0.20 & 0.19 & 0.05 & 0.09 & 0.08 & 0.17 & - & 0.65 & - & 0.13 & 0.08 & 0.11 & 0.07 & 0.13 & 0.11 & - & - & - & - & - \\
\hline & & & & (4) & (4) & (2) & (4) & (2) & (9) & (2) & (13) & (2) & (15) & - & (2) & - & (13) & (2) & (15) & (2) & (77) & (5) & - & (1) & (1) & - & (1) \\
\hline 50252 & 10600 & 3.7 & 195 & 11.30 & - & - & 8.14 & - & 7.21 & - & 6.54 & - & - & - & - & - & 4.08 & - & - & - & 6.61 & - & - & - & - & - & - \\
\hline & & & 10 & 0.06 & - & - & - & - & 0.79 & - & 0.29 & - & - & - & - & - & 0.72 & - & - & - & 0.11 & - & - & - & & - & - \\
\hline & & & & (2) & - & - & (1) & - & (2) & - & (2) & - & - & - & - & - & (2) & - & - & - & (4) & - & - & - & - & - & - \\
\hline 50513 & 11200 & 4.0 & 121 & 11.03 & - & - & 8.28 & - & 7.00 & - & 6.71 & 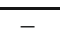 & - & - & - & - & 4.33 & - & - & - & 6.71 & - & - & - & & 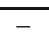 & - \\
\hline & & & 6 & 0.04 & - & - & - & - & 0.08 & - & 0.05 & - & - & - & - & - & 0.67 & - & - & - & 0.15 & - & - & - & & - & - \\
\hline & & & & (2) & - & - & (1) & - & (2) & - & (2) & - & - & $\begin{array}{lll}- & \\
-\end{array}$ & - & - & (2) & - & - & - & (3) & - & - & - & - & - & - \\
\hline 50751 & 13500 & 3.8 & 243 & 11.10 & - & - & - & - & 7.61 & - & 6.46 & - & 6.46 & - & - & - & - & - & - & - & 5.66 & - & - & - & & - & - \\
\hline & & & 14 & 0.07 & - & - & - & - & 0.05 & - & 0.13 & - & 0.31 & _- & - & 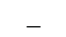 & - & - & - & - & 0.04 & - & - & - & & _- & - \\
\hline & & & & (6) & - & - & - & - & (2) & _- & (2) & - & (2) & - & - & - & - & - & - & - & (2) & - & - & - & - & - & _- \\
\hline 51079 & 11700 & 3.2 & 161 & 11.06 & - & - & - & - & 7.74 & - & 6.95 & - & 6.35 & - & - & - & 4.17 & - & 6.54 & - & 7.10 & - & - & - & & - & - \\
\hline & & & 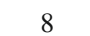 & 0.02 & - & - & _- & - & - & - & 0.30 & - & 0.39 & - & - & - & 0.35 & - & 0.16 & - & 0.11 & - & - & - & & _ & - \\
\hline & & & & (3) & - & - & - & - & (1) & - & (2) & - & (2) & - & - & - & (2) & - & (2) & - & (3) & - & - & - & - & - & - \\
\hline 52312 & 13000 & 3.4 & 175 & 10.74 & - & - & 9.05 & - & 6.55 & - & 7.31 & - & 7.31 & - & 6.76 & - & 5.47 & - & 6.40 & - & 7.40 & - & - & - & - & - & - \\
\hline & & & 10 & 0.04 & - & - & 0.20 & - & 0.01 & 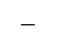 & 0.05 & - & - & - & - & - & 0.47 & - & - & - & 0.13 & - & - & - & & - & - \\
\hline & & & & (2) & - & - & (2) & - & (2) & - & (2) & - & (1) & - & (1) & - & (2) & - & (1) & - & (8) & - & - & - & - & - & - \\
\hline 53004 & 11600 & 4.0 & 58 & 10.78 & 8.21 & - & 8.82 & - & 7.58 & - & 7.13 & - & 7.13 & - & - & 2.92 & 5.08 & - & 5.94 & 7.62 & 7.03 & - & - & - & - & - & - \\
\hline & & & 8 & 0.03 & - & - & - & - & 0.03 & - & 0.06 & - & 0.11 & - & - & - & 0.14 & - & 0.05 & - & 0.14 & - & - & - & - & - & - \\
\hline & & & & (2) & (1) & - & (1) & - & (2) & - & (4) & - & (2) & - & - & (1) & (8) & - & (4) & (1) & (11) & - & - & - & - & - & - \\
\hline 53083 & 11600 & 3.5 & 90 & 10.93 & - & - & 8.86 & - & 7.27 & - & 6.89 & - & 7.21 & - & - & - & 4.55 & - & 5.27 & - & 6.97 & - & - & - & - & - & - \\
\hline & & & 7 & 0.10 & - & - & 0.03 & - & 0.03 & - & 0.15 & - & 0.80 & - & - & - & 0.03 & - & 0.20 & - & 0.15 & - & - & - & - & - & - \\
\hline & & & & (5) & - & - & (2) & - & (2) & - & (3) & - & (2) & - & - & - & (3) & - & (3) & - & (10) & - & - & - & - & - & - \\
\hline 53851 & 13600 & 3.7 & 40 & 10.36 & 7.74 & - & - & - & 5.77 & - & 7.71 & - & 6.20 & - & - & - & 5.16 & - & 5.87 & - & 7.55 & - & - & 3.61 & - & - & - \\
\hline & & & 3 & 0.09 & - & - & - & - & - & - & 0.13 & - & 0.19 & - & - & - & 0.08 & - & 0.15 & - & 0.19 & - & - & 0.13 & - & - & - \\
\hline & & & & (3) & (1) & - & - & - & (1) & - & (8) & - & (4) & - & - & - & (2) & - & (5) & - & (31) & - & - & (2) & - & - & - \\
\hline 54929 & 10000 & 3.3 & 81 & 11.07 & - & - & 8.78 & - & 7.24 & 6.41 & 7.27 & - & - & - & 6.10 & - & 4.47 & - & 5.47 & 4.85 & 7.01 & - & - & - & - & - & $\overline{2.23}$ \\
\hline & & & 3 & 0.01 & - & - & 0.01 & - & 0.06 & - & 0.03 & - & - & - & - & - & 0.16 & - & 0.06 & - & 0.11 & - & - & - & - & - & - \\
\hline & & & & (2) & - & - & (2) & - & (4) & (1) & (3) & - & - & - & (1) & - & (12) & - & (6) & (1) & (15) & - & - & - & - & - & (1) \\
\hline 55362 & 13000 & 4.0 & 53 & 10.40 & - & - & - & - & 5.84 & - & 6.02 & - & 5.18 & - & - & - & 5.85 & - & 5.68 & 6.24 & 6.99 & - & - & - & - & - & - \\
\hline & & & 6 & 0.10 & - & - & - & - & - & - & 0.01 & - & 0.51 & - & - & - & 0.38 & - & 0.22 & 0.07 & 0.19 & - & - & - & - & - & - \\
\hline & & & & (2) & - & - & - & - & (1) & - & (2) & - & (2) & - & - & - & (2) & - & (2) & (3) & (13) & - & - & - & - & - & - \\
\hline 55793 & 11900 & 3.5 & 108 & 11.05 & - & - & 8.75 & - & 7.68 & - & 7.06 & - & 6.97 & - & - & - & 4.34 & 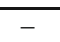 & 5.45 & - & 7.04 & - & - & - & & - & - \\
\hline & & & 7 & 0.07 & - & - & - & - & 0.14 & - & 0.08 & - & 0.06 & - & - & - & 0.25 & - & 0.12 & - & 0.18 & - & - & 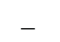 & - & - & - \\
\hline & & & & (5) & - & - & (1) & - & (3) & - & (3) & - & (2) & - & - & - & (2) & - & (2) & - & (11) & - & - & - & - & - & - \\
\hline 56006 & 14800 & 3.7 & 203 & 11.20 & 8.34 & - & - & 8.06 & 7.60 & - & 7.53 & - & 7.08 & - & 4.47 & 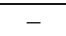 & - & - & - & - & 7.96 & 7.00 & - & - & & - & - \\
\hline & & & 10 & 0.07 & 0.08 & - & - & - & 0.08 & - & 0.31 & - & 0.03 & - & - & - & - & - & - & - & 0.80 & - & - & - & - & - & - \\
\hline & & & & (7) & (2 & - & - & (1 & (2) & - & (2) & - & (2) & - & (1) & - & - & - & - & - & (2) & (1) & - & - & - & - & - \\
\hline 56446 & 12800 & 3.5 & 195 & 11.03 & 8.53 & - & - & 7.77 & 7.20 & - & 7.03 & - & 7.35 & - & - & - & 5.54 & - & - & - & 7.11 & - & - & - & & - & - \\
\hline & & & 15 & 0.05 & - & - & - & 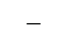 & 0.83 & - & 0.01 & - & 0.11 & - & - & - & - & - & - & - & 0.16 & - & - & - & 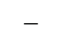 & - & - \\
\hline & & & & (2) & - & - & - & (1) & (2) & - & (2) & - & (3) & - & - & - & (1) & - & - & - & (4) & - & - & - & - & - & - \\
\hline 56610 & 13700 & 3.7 & 100 & 10.29 & - & - & - & - & 6.84 & - & 7.47 & - & 7.17 & - & - & - & 5.72 & - & 6.38 & - & 8.07 & - & - & - & & - & - \\
\hline & & & 10 & 0.03 & - & - & - & - & - & - & 0.14 & - & 0.09 & - & - & - & 0.18 & - & 0.16 & - & 0.14 & - & - & - & - & _- & - \\
\hline & & & & (3) & - & - & - & - & (1) & - & (6) & - & (2) & - & - & - & (4) & - & (4) & - & (13) & - & - & - & - & - & - \\
\hline 5613 & 13300 & 4.0 & 97 & 11.00 & 8.04 & - & - & - & 7.66 & - & 7.28 & - & 7.11 & - & 5.98 & - & 5.08 & - & - & - & 7.27 & 6.22 & - & - & - & - & - \\
\hline & & & 8 & 0.07 & - & - & - & - & 0.25 & - & 0.15 & - & 0.13 & - & - & - & 0.74 & - & - & - & 0.09 & - & - & - & - & - & - \\
\hline & & & & (3) & (1) & - & - & - & (2) & - & (2) & - & (4) & - & (1) & - & (2) & - & - & - & (5) & (1) & - & - & - & - & - \\
\hline 57275 & 9600 & 3.5 & 132 & 11.05 & 7.73 & - & 8.65 & - & 6.99 & - & 6.98 & - & - & - & 6.14 & - & 4.45 & - & - & - & 6.97 & - & - & - & - & - & - \\
\hline & & & 9 & - & - & - & - & - & 0.07 & - & 0.25 & - & - & - & - & - & 0.03 & - & - & - & 0.18 & - & - & - & - & - & - \\
\hline & & & & (1) & (1) & - & (1) & - & (2) & - & (2) & - & - & - & (1) & - & (3) & - & - & - & (7) & - & - & - & - & - & - \\
\hline 57293 & 12000 & 4.0 & 160 & 11.04 & - & - & 8.62 & - & 8.16 & - & 7.47 & - & 8.02 & - & - & - & 5.34 & - & 6.38 & - & 7.35 & - & - & - & - & - & - \\
\hline & & & 17 & 0.32 & - & - & - & - & 0.56 & - & 0.23 & - & - & - & - & - & 0.04 & - & 0.12 & - & 0.07 & - & - & - & - & - & - \\
\hline & & & & (2) & - & - & (1) & - & (2) & - & (2) & - & (1) & - & - & - & (2) & - & (2) & - & (4) & - & - & - & - & - & - \\
\hline
\end{tabular}


Table 2. continued.

\begin{tabular}{|c|c|c|c|c|c|c|c|c|c|c|c|c|c|c|c|c|c|c|c|c|c|c|c|c|c|c|c|}
\hline HD & $\begin{array}{l}T_{\text {eff }} \\
{[\mathrm{K}]}\end{array}$ & $g g$ & $\begin{array}{r}V \sin i \\
\mathrm{~km} \mathrm{~s}^{-1}\end{array}$ & $\mathrm{He}$ & $\bar{C}$ & $\mathrm{~N}$ & $\mathrm{O}$ & $\mathrm{Ne}$ & $\mathrm{Mg}$ & $\overline{\mathrm{Al}}$ & $\mathrm{Si}$ & $\overline{\mathrm{P}}$ & $\overline{\mathrm{S}}$ & $\overline{\mathrm{Cl}}$ & $\overline{\mathrm{Ca}}$ & $\overline{\mathrm{Sc}}$ & $\overline{\mathrm{Ti}}$ & $\overline{\mathrm{V}}$ & $\overline{\mathrm{Cr}}$ & $\overline{\mathrm{Mn}}$ & $\overline{\mathrm{Fe}}$ & $\overline{\mathrm{Ni}}$ & $\overline{\mathrm{Ga}}$ & $\overline{\mathrm{Sr}}$ & $\bar{Y}$ & $\overline{\mathrm{Zr}}$ & $\overline{\mathrm{Ba}}$ \\
\hline \multirow[t]{3}{*}{168202} & 10500 & 4.0 & 71 & 11.01 & - & - & 8.84 & - & 7.55 & - & 7.19 & - & - & - & 6.66 & 3.01 & 5.01 & - & 5.92 & 6.27 & 7.44 & - & - & - & - & - & 3.39 \\
\hline & & & 6 & - & - & - & 0.24 & - & 0.01 & - & 0.01 & - & - & - & - & - & 0.10 & - & 0.11 & - & 0.17 & - & - & - & - & - & - \\
\hline & & & & (1) & - & - & (2) & - & (2) & - & (2) & - & - & - & (1) & (1) & (9) & - & (6) & (1) & (17) & - & - & - & - & - & (1) \\
\hline \multirow[t]{3}{*}{168932} & 10000 & 4.0 & 38 & 10.26 & 8.28 & 8.85 & 8.48 & - & 7.93 & 6.75 & 7.52 & - & - & - & 6.34 & 2.43 & 5.25 & 4.67 & 6.32 & 6.24 & 7.98 & - & - & 4.29 & 3.00 & 3.46 & 3.73 \\
\hline & & & 1 & 0.12 & 0.08 & - & 0.04 & - & 0.12 & - & 0.20 & - & - & - & 0.14 & 0.06 & 0.15 & - & 0.07 & 0.10 & 0.08 & - & - & - & 0.02 & - & 0.10 \\
\hline & & & & (3) & (2) & (1) & (2) & - & (6) & (1) & (3) & - & - & - & (2) & (2) & (20) & (1) & (11) & (4) & (42) & - & - & (1) & (2) & (1) & (3) \\
\hline \multirow[t]{3}{*}{169224} & 11200 & 4.0 & 152 & 11.01 & - & - & 8.77 & - & 7.68 & - & 7.21 & - & 7.15 & - & - & - & 4.70 & - & 5.55 & - & 7.19 & - & - & - & - & - & - \\
\hline & & & 11 & - & - & - & - & - & - & - & 0.12 & - & - & - & - & - & 0.17 & - & 0.07 & - & 0.05 & - & - & - & - & - & - \\
\hline & & & & (1) & - & - & (1) & - & (1) & - & (2) & - & (1) & - & - & - & (2) & - & (2) & - & (3) & - & - & - & - & - & - \\
\hline 169225 & 10100 & 3.7 & 182 & 10.92 & - & - & - & - & 7.86 & - & 7.51 & - & - & - & 7.11 & - & 4.74 & - & 5.42 & - & 7.37 & - & - & - & - & - & 2.83 \\
\hline & & & 4 & - & - & - & - & - & - & - & - & - & - & - & - & - & 0.14 & - & 0.38 & - & 0.12 & - & - & - & - & - & - \\
\hline & & & & (1) & - & - & - & - & (1) & - & (1) & - & - & - & (1) & - & (3) & - & (2) & - & (3) & - & - & - & - & - & (1) \\
\hline 169512 & 12000 & 3.8 & 231 & 10.98 & - & - & - & - & 7.99 & - & 7.38 & - & - & - & - & - & 4.79 & - & 5.19 & - & 7.38 & - & - & - & - & - & - \\
\hline & & & 12 & - & - & - & - & - & 0.15 & - & - & - & - & - & - & - & - & - & - & - & 0.14 & - & - & - & - & - & - \\
\hline & & & & (1) & - & - & - & - & (2) & - & (1) & - & - & - & - & - & (1) & - & (1) & - & (2) & - & - & - & - & - & - \\
\hline 169578 & 12000 & 3.7 & 259 & 11.09 & - & - & - & - & 8.24 & - & 7.52 & - & - & - & - & - & 4.88 & - & 5.28 & - & 7.16 & - & - & - & - & - & - \\
\hline & & & 14 & 0.10 & - & - & - & - & 0.09 & - & 0.23 & - & - & - & - & - & 0.43 & - & - & - & 0.04 & - & - & - & - & - & - \\
\hline & & & & (2) & - & - & - & - & (2) & - & (2) & - & - & - & - & - & (2) & - & (1) & - & (3) & - & - & - & - & - & - \\
\hline 170783 & 13800 & 3.0 & 295 & 11.11 & - & - & - & - & 8.06 & - & 7.62 & - & 7.34 & - & - & - & - & - & - & - & 6.99 & - & - & - & - & - & - \\
\hline & & & 11 & 0.06 & - & - & - & - & 0.46 & - & - & - & 0.23 & - & - & - & - & - & - & - & - & - & - & - & - & - & - \\
\hline & & & & (2) & - & - & - & - & (2) & - & (1) & - & (2) & - & - & - & - & - & - & - & (1) & - & - & - & - & - & - \\
\hline 170935 & 10300 & 3.0 & 271 & 10.79 & - & - & - & - & 8.18 & - & 7.36 & - & - & - & - & - & 4.64 & - & 5.66 & - & 7.57 & - & - & - & - & - & - \\
\hline & & & 16 & - & - & - & - & - & 0.09 & - & - & - & - & - & - & - & - & - & - & - & 0.03 & - & - & - & - & - & - \\
\hline & & & & (1) & - & - & - & - & (2) & - & (1) & - & - & - & - & - & (1) & - & (1) & - & (2) & - & - & - & - & - & - \\
\hline 171247 & 12300 & 3.5 & 68 & - & - & - & - & - & 7.48 & - & 8.32 & - & 6.75 & - & - & - & 5.00 & - & 6.05 & 6.85 & 7.72 & - & - & - & - & - & - \\
\hline & & & 2 & - & - & - & - & - & 0.09 & - & 0.12 & - & - & - & - & - & 0.12 & - & 0.03 & - & 0.13 & - & - & - & - & - & - \\
\hline & & & & - & - & - & - & - & (4) & - & (5) & - & (1) & - & - & - & (5) & - & (6) & (1) & (18) & - & - & - & - & - & - \\
\hline 171931 & 13000 & 3.7 & 283 & 10.93 & - & - & - & - & 7.91 & - & 6.82 & - & 7.79 & - & - & - & 5.71 & - & 5.57 & - & 6.94 & 7.67 & - & - & - & - & - \\
\hline & & & 30 & 0.02 & - & - & - & - & 0.01 & - & - & - & 0.65 & - & - & - & 0.34 & - & 0.32 & - & - & - & - & - & - & . & - \\
\hline & & & & (2) & - & - & - & - & (2) & - & (1) & - & (2) & - & - & - & (2) & - & (2) & - & (1) & (1) & - & - & - & - & - \\
\hline 172850 & 12000 & 3.9 & 84 & 10.99 & - & - & 8.66 & - & 7.17 & - & 6.81 & - & - & - & 6.80 & - & 4.70 & - & 5.43 & - & 6.89 & - & - & - & - & - & - \\
\hline & & & 8 & 0.08 & - & - & 0.07 & - & 0.12 & - & 0.07 & - & - & - & - & - & 0.29 & - & 0.02 & - & 0.15 & - & - & - & - & - & - \\
\hline & & & & (5) & - & - & (2) & - & (4) & - & (5) & - & - & - & (1) & - & (4) & - & (2) & - & (16) & - & - & - & - & - & - \\
\hline 173673 & 13000 & 3.8 & 29 & - & 7.92 & - & - & - & 6.90 & 6.84 & 7.25 & 6.90 & 6.73 & - & - & - & 5.73 & - & 6.08 & - & 8.06 & 6.20 & - & - & - & - & - \\
\hline & & & 2 & - & - & - & - & - & 0.16 & - & 0.06 & 0.04 & 0.08 & - & - & - & 0.18 & - & 0.01 & - & 0.13 & - & - & - & - & - & - \\
\hline & & & & - & (1) & - & - & - & (2) & (1) & (6) & (2) & (3) & - & - & - & (6) & - & (3) & - & (50) & (1) & - & - & - & - & - \\
\hline 174701 & 13500 & 3.9 & 178 & 11.13 & - & - & 9.21 & - & 7.83 & - & 7.51 & - & 7.22 & - & - & - & 5.21 & - & - & - & 7.69 & - & - & - & - & - & - \\
\hline & & & 8 & 0.03 & - & - & - & - & - & - & 0.04 & - & - & - & - & - & 0.40 & - & - & - & 0.11 & - & - & - & - & - & - \\
\hline & & & & (2) & - & - & (1) & - & (1) & - & (2) & - & (1) & - & - & - & (2) & - & - & - & (2) & - & - & - & - & - & - \\
\hline 174836 & 10300 & 4.3 & 149 & - & - & - & 9.02 & - & 7.87 & - & 7.24 & - & - & - & 6.88 & - & 5.08 & - & 5.65 & - & 7.63 & - & - & - & - & - & 3.49 \\
\hline & & & 8 & - & - & - & - & - & - & - & 0.01 & - & - & - & - & - & 0.16 & - & 0.06 & - & 0.07 & - & - & - & - & - & - \\
\hline & & & & - & - & - & (1) & - & (1) & - & (2) & - & - & - & (1) & - & (4) & - & (3) & - & (2) & - & - & - & - & - & (1) \\
\hline 174884 & 13200 & 3.6 & 113 & 11.04 & 8.26 & - & - & - & 7.81 & - & 6.98 & - & 7.02 & - & - & - & 4.65 & - & 5.66 & - & 7.09 & - & - & - & - & - & - \\
\hline & & & 4 & 0.10 & - & - & - & - & 0.04 & - & 0.52 & - & 0.14 & - & - & - & 0.13 & - & 0.14 & - & 0.12 & - & - & - & - & - & - \\
\hline & & & & (4) & (1) & - & - & - & (2) & - & (2) & - & (3) & - & - & - & (2) & - & (2) & - & (6) & - & - & - & - & - & - \\
\hline 176076 & 10400 & 3.7 & 42 & 10.87 & - & - & 8.79 & - & 7.56 & - & 7.32 & - & - & - & 6.33 & 3.08 & 4.90 & - & 5.72 & 4.68 & 7.28 & 6.61 & - & 3.10 & - & - & 3.16 \\
\hline & & & 2 & 0.11 & - & - & 0.01 & - & 0.15 & - & 0.07 & - & - & - & 0.21 & 0.21 & 0.11 & - & 0.16 & - & 0.12 & 0.02 & - & - & - & - & - \\
\hline & & & & (2) & - & - & (2) & - & (4) & - & (8) & - & - & - & (2) & (4) & (14) & - & (11) & (1) & (26) & (2) & - & (1) & - & - & (1) \\
\hline 176158 & 14500 & 3.7 & 126 & 11.12 & 8.22 & - & - & - & 7.06 & - & 6.89 & - & 6.61 & - & 7.42 & - & 5.78 & - & 6.49 & - & 6.41 & - & - & - & - & - & - \\
\hline & & & 5 & 0.13 & - & - & - & - & - & - & 0.08 & - & - & - & - & - & - & - & - & - & 0.14 & - & - & - & - & - & - \\
\hline & & & & (4) & (1) & - & - & - & (1) & - & (2) & - & (1) & - & (1) & - & (1) & - & (1) & - & (2) & - & - & - & - & - & - \\
\hline 176258 & 11000 & 3.9 & 303 & 11.00 & - & - & - & - & 7.92 & - & - & - & - & - & - & - & 5.03 & - & - & - & 7.47 & - & - & - & - & - & - \\
\hline & & & 8 & 0.13 & - & - & - & - & 0.01 & - & - & - & - & - & - & - & 0.07 & - & - & - & - & - & - & - & - & - & - \\
\hline & & & & (2) & - & - & - & - & (2) & - & - & - & - & - & - & - & (2) & - & - & - & (1) & - & - & - & - & - & - \\
\hline 177756 & 11700 & 4.0 & 177 & 10.91 & - & - & - & - & 7.57 & - & 7.64 & - & - & - & - & - & 4.42 & - & 5.82 & - & 7.18 & - & - & - & - & - & - \\
\hline & & & 6 & 0.09 & - & - & - & - & - & - & - & - & - & - & - & - & 0.02 & - & 0.60 & - & 0.09 & - & - & - & - & - & - \\
\hline & & & & (2) & - & - & - & - & (1) & - & (1) & - & - & - & - & - & (2) & - & (2) & - & (3) & - & - & - & - & - & - \\
\hline 177880 & 14700 & 3.8 & 56 & 11.03 & 8.39 & - & - & 8.42 & 7.67 & - & 7.03 & - & 7.05 & - & - & - & - & - & - & - & 7.14 & - & - & - & - & - & - \\
\hline & & & 4 & 0.03 & - & - & - & 0.06 & 0.29 & - & 0.12 & - & 0.13 & - & - & - & - & - & - & - & 0.10 & - & - & - & - & - & - \\
\hline & & & & $(5)$ & (1) & - & - & (2) & (2) & - & (4) & - & (6) & - & - & - & - & - & - & - & (5) & - & - & - & - & - & - \\
\hline 178744 & 14500 & 3.5 & 220 & 11.12 & - & - & - & - & 7.92 & - & 7.24 & - & 7.47 & - & - & - & 5.76 & - & - & - & 6.51 & - & - & - & - & - & - \\
\hline & & & 8 & 0.03 & - & - & - & - & - & - & 0.75 & - & - & - & - & - & - & - & - & - & - & - & - & - & - & - & - \\
\hline & & & & (2) & - & - & - & - & (1) & - & (2) & - & (1) & - & - & - & (1) & - & - & - & (1) & - & - & - & - & - & - \\
\hline
\end{tabular}


Table 2. continued.

\begin{tabular}{|c|c|c|c|c|c|c|c|c|c|c|c|c|c|c|c|c|c|c|c|c|c|c|c|c|c|c|c|}
\hline HD & $\begin{array}{l}T_{\text {eff }} \\
{[\mathrm{K}]}\end{array}$ & $\overline{\log g}$ & $\begin{array}{c}V \sin i \\
\mathrm{~km} \mathrm{~s}^{-1}\end{array}$ & $\overline{\mathrm{He}}$ & $\overline{\mathrm{C}}$ & $\overline{\overline{\mathrm{N}}}$ & $\overline{\mathrm{O}}$ & $\overline{\mathrm{Ne}}$ & $\overline{\mathrm{Mg}}$ & $\overline{\mathrm{Al}}$ & $\overline{\overline{\mathrm{Si}}}$ & $\overline{\overline{\mathrm{P}}}$ & $\overline{\mathrm{S}}$ & $\overline{\mathrm{Cl}}$ & $\overline{\mathrm{Ca}}$ & $\overline{\mathrm{Sc}}$ & $\overline{\mathrm{Ti}}$ & $\overline{\mathrm{V}}$ & $\overline{\mathrm{Cr}}$ & $\overline{\mathrm{Mn}}$ & $\overline{\mathrm{Fe}}$ & $\overline{\mathrm{Ni}}$ & $\overline{\mathrm{Ga}}$ & $\overline{\mathrm{Sr}}$ & $\overline{\mathrm{Y}}$ & $\overline{\mathrm{Zr}}$ & $\overline{\overline{\mathrm{Ba}}}$ \\
\hline \multirow{3}{*}{179124} & 11600 & 3.2 & 298 & 11.23 & - & - & - & - & 8.40 & - & 7.55 & - & - & - & - & - & 4.23 & - & - & - & 6.22 & - & - & - & - & - & - \\
\hline & & & 5 & 0.08 & - & - & - & - & - & - & - & - & - & - & - & - & - & - & - & - & 0.10 & - & - & - & - & - & - \\
\hline & & & & (2) & - & - & - & - & (1) & - & (1) & - & - & - & - & - & (1) & - & - & - & (2) & - & - & - & - & - & - \\
\hline \multirow[t]{3}{*}{179761} & 12800 & 3.4 & 17 & 11.15 & 8.28 & 8.30 & 8.85 & 8.00 & 7.40 & - & 7.42 & - & 6.97 & - & 6.41 & - & 4.61 & - & 5.54 & 6.26 & 7.30 & 6.07 & - & 2.76 & - & - & - \\
\hline & & & 1 & 0.09 & - & - & 0.15 & - & 0.06 & - & 0.13 & - & 0.17 & - & 0.22 & - & 0.14 & - & 0.07 & - & 0.10 & 0.20 & - & - & - & - & - \\
\hline & & & & (5) & (1) & (1) & (2) & (1) & (5) & - & (8) & - & (10) & - & (2) & - & (7) & - & (9) & (1) & (44) & (5) & - & (1) & - & - & - \\
\hline \multirow[t]{3}{*}{181440} & 11200 & 3.5 & 58 & 10.90 & - & - & - & - & 7.80 & - & 7.24 & - & - & - & - & - & 4.36 & - & 5.09 & - & 6.85 & - & - & - & - & - & - \\
\hline & & & 1 & 0.12 & - & - & - & - & 0.26 & - & 0.59 & - & - & - & - & - & 0.45 & - & - & - & 0.08 & - & - & - & - & - & - \\
\hline & & & & (2) & - & - & - & - & (2) & - & (2) & - & - & - & - & - & (2) & - & (1) & - & (5) & - & - & - & - & - & - \\
\hline \multirow[t]{3}{*}{181761} & 14600 & 3.6 & 35 & 10.91 & 7.61 & - & 8.49 & 7.68 & 7.17 & - & 6.45 & - & 6.99 & - & - & - & 5.45 & - & 5.23 & - & 7.12 & - & - & - & - & - & - \\
\hline & & & 3 & 0.11 & - & - & - & - & 0.30 & - & 0.19 & - & 0.01 & - & - & - & 0.04 & - & - & - & 0.17 & - & - & - & - & - & - \\
\hline & & & & (4) & (1) & - & (1) & (1) & (2) & - & (3) & - & (2) & - & - & - & (2) & - & (1) & - & (5) & - & - & - & - & - & - \\
\hline \multirow[t]{3}{*}{182198} & 11450 & 3.5 & 25 & 10.93 & 8.38 & - & 7.61 & - & 7.48 & 6.56 & 7.34 & 5.28 & 7.06 & - & 6.15 & - & 4.61 & 4.55 & 5.65 & - & 7.27 & 6.45 & - & - & - & -2 & $\overline{2.56}$ \\
\hline & & & 1 & 0.03 & 0.14 & - & 0.24 & - & 0.13 & 0.42 & 0.09 & 0.02 & 0.17 & - & 0.12 & - & 0.14 & 0.01 & 0.12 & - & 0.16 & 0.12 & - & - & - & & 0.01 \\
\hline & & & & (5) & (8) & - & (2) & - & (22) & (6) & (14) & (2) & (20) & - & (5) & - & (18) & (2) & (30) & - & (93) & (15) & - & - & - & & (2) \\
\hline
\end{tabular}

